\title{
Noiseless Vlasov-Poisson simulations with linearly transformed particles
}

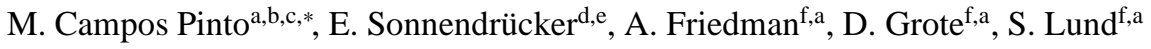 \\ ${ }^{a}$ Lawrence Berkeley National Laboratory, Berkeley, CA 94720, USA \\ ${ }^{b}$ CNRS, UMR 7598, Laboratoire Jacques-Louis Lions, F-75005, Paris, France \\ ${ }^{c}$ UPMC Univ Paris 06, UMR 7598, Laboratoire Jacques-Louis Lions, F-75005, Paris, France \\ ${ }^{d}$ IRMA, UMR 7501, Université de Strasbourg E CNRS, 7 rue René Descartes, F-67084 Strasbourg Cedex, France \\ ${ }^{e}$ Project-team CALVI, INRIA Nancy Grand Est, 7 rue René Descartes, F-67084 Strasbourg Cedex, France \\ ${ }^{f}$ Lawrence Livermore National Laboratory, Livermore, CA 94550, USA
}

\begin{abstract}
We introduce a deterministic discrete-particle simulation approach, the Linearly-Transformed Particle-In-Cell (LTPIC) method, that employs linear deformations of the particles to reduce the noise traditionally associated with particle schemes. Formally, transforming the particles is justified by local first order expansions of the characteristic flow in phase space. In practice the method amounts to using deformation matrices within the particle shape functions; these matrices are updated via local evaluations of the forward numerical flow. Because it is necessary to periodically remap the particles on a regular grid to avoid excessively deforming their shapes, the method can be seen as a development of Denavit's Forward Semi-Lagrangian (FSL) scheme [J. Denavit, J. Comp. Physics 9, 75 (1972)]. However, it has recently been established [M. Campos Pinto, "Smooth particle methods without smoothing", arXiv:1112.1859 (2012)] that the underlying Linearly-Transformed Particle scheme converges for abstract transport problems, with no need to remap the particles; deforming the particles can thus be seen as a way to significantly lower the remapping frequency needed in the FSL schemes, and hence the associated numerical diffusion. To couple the method with electrostatic field solvers, two specific charge deposition schemes are examined, and their performance compared with that of the standard deposition method. Finally, numerical $1 \mathrm{~d} 1 \mathrm{v}$ simulations involving benchmark test cases and halo formation in an initially mismatched thermal sheet beam demonstrate some advantages of our LTPIC scheme over the classical PIC and FSL methods. Benchmarked test cases also indicate that, for numerical choices involving similar computational effort, the LTPIC method is capable of accuracy comparable to or exceeding that of state-of-the-art, high-resolution Vlasov schemes.
\end{abstract}

Keywords: particle method, Vlasov-Poisson equation, plasma, noiseless method, remapping, beam halo simulations

*Corresponding author. LJLL, Université Pierre et Marie Curie, Boîte courrier 187, 75252 Paris Cedex 05, France. Tel.: +331442791 54; fax: +3314427 7200 .

Email addresses: campos@ann. jussieu.fr (M. Campos Pinto), sonnen@math. unistra.fr (E. Sonnendrücker), af@llnl.gov (A. Friedman), grote1@llnl.gov (D. Grote), smlund@llnl.gov (S. Lund) 


\section{Introduction}

Although considered very efficient in many practical cases, particle-in-cell (PIC) simulations sometimes present levels of noise that make fine plasma phenomena very numerically expensive to resolve. The fact that particles are usually initialized with random procedures explains part of the statistical noise, yet there is another reason for the birth of strong oscillations in the numerical solutions. Indeed, it is known from the mathematical analysis of deterministic particle methods [1, 2] that a typical requirement for smooth convergence is that the radius $\varepsilon$ of the particles tend to 0 at a much slower rate than the average grid spacing $h$ used for their initialization, a property that is expensive to satisfy in practice. Here by "smooth convergence" we mean the pointwise convergence of the density function carried by the macro-particles, towards the exact solution $f$ of the Vlasov equation. If the latter is a continuous function of the phase-space coordinates and if the convergence is pointwise, numerical solutions are indeed free of spurious oscillations, at least asymptotically.

Specifically, smooth convergence requires $\varepsilon \sim h^{q}$ with $q<1$, which can be interpreted as an extended overlapping condition: as the initialization grid gets finer, more and more particles must overlap. In PIC schemes (and more precisely, weighted PIC schemes with uniform Poissonsolver grids) the particle size is implicitly dictated by the $d$-dimensional mesh used in the field solver through its number of cells $N_{c} \sim \varepsilon^{-d}$, whereas the (average, if random) initial spacing can be derived from the number of particles $N_{\mathrm{p}} \sim h^{-d-d^{\prime}}$, with $d^{\prime}$ denoting the dimension of the velocity variable. Therefore, to guarantee the smooth convergence of the numerical density one should increase the number of particles per cell consistent with the number of cells, i.e.,

$$
\frac{N_{\mathrm{p}}}{N_{\mathrm{c}}} \sim N_{\mathrm{c}}^{\frac{d+d^{\prime}}{d q}-1} .
$$

Here the exponent is always positive, and when $d=d^{\prime}$ it is greater than unity, e.g., 1.5 for $q=0.8$. Hence for smooth particle simulations, the number of particles per cell should increase significantly faster than the number of cells. In practice such a condition is usually not met.

On the mathematical level, particle methods that do not meet the extended overlapping condition may still converge towards a smooth $f$ but only in a weak sense, i.e., in the sense that the local integrals of the particle density function tend to the same integrals of $f$. This case typically corresponds to simulations with strongly oscillating density functions, where accurate results can be obtained for certain integral quantities such as the electric field, or for the density itself, through appropriate smoothing procedures. And since the accuracy of the electric field is what matters most for the (electrostatic) dynamics of the system, strongly oscillating simulations can very well give satisfactory results on the longer scale sizes of physical interest.

However, in cases where the physics of interest is in a region of low plasma density, smooth convergence seems to be necessary for precise measurements. For a variety of practical problems indeed (including backward Raman scattering [3], plasma-wall transitions [4, 5], halo formation in beams [6] and development of electron holes in the presence of a guide field [7]) physicists often need to resort to (grid based) Vlasov or Semi-Lagrangian solvers in order to obtain sufficient accuracy. Unfortunately these methods are known to be numerically expensive to run and challenging to implement, as they require the mesh to cover the whole phase space and can suffer from diffusive effects.

To reduce noise, Denavit [8] proposed a particle method later revisited as a Forward SemiLagrangian (FSL) scheme [9, 10], where the distribution function carried by the particles is periodically remapped to the nodes of a phase-space grid. This has a smoothing effect which 
in practice eliminates the need for extended overlaping. However, frequent remappings can introduce unwanted numerical diffusion which in many cases contradicts the benefit of using lowdiffusion particle schemes. Resulting numerical diffusion from the remappings can be reduced by use of high order adaptive schemes; see, e.g., [11, 12]. Other methods to reduce the noise have also been studied, such as wavelet-based denoising techniques; see e.g., [13, 14, 15].

In this article, we present a new particle scheme where in addition to pushing the particle centers along their trajectories, one updates the particle shapes through the use of local linear transformations to better follow the local shear and rotation flows in phase space. As in the FSL scheme, the method is purely deterministic, and to prevent particles from being arbitrarily stretched, the particles need to be remapped periodically. However, significantly lower remapping frequencies are needed in practice, which results in higher accuracy and less numerical diffusion. On a theoretical level this advantage is supported by the fact that for transport problems with prescribed characteristic flow, the linearly transformed particle solutions are shown to converge in the uniform norm as $h$ tends to 0 , without any remappings; see [16].

Deforming the particles is not a new idea. For instance, our scheme can be viewed as a variation on Hou's formal vortex method [17] where the particles are deformed through a global mapping. In our method, each particle is transported by the linearized flow around its trajectory. Still on a formal level this approach coincides with a method presented by Cohen and Perthame [18] who established its first-order convergence, but they did not provide a numerical scheme to compute the deformation matrices. In the context of plasma simulation, an important class of deformed particle methods is offered by the Complex Particle Kinetic (CPK) schemes introduced by Bateson and Hewett [19, 20]. In the CPK method, in addition to having the Gaussian shape of the particles transformed by the local shearing of the flow, the particles can also be fragmented to probe for emerging features, and merged where fine particles are no longer needed. Another exciting method is the Cloud in Mesh (CM) scheme of Alard and Colombi [21] that has been brought to our attention after the writing of this article. CM particles have Gaussian shapes as in the CPK method, and they are deformed by local linearizations of the force field, in a manner similar to ours. Moreover, in [21] the authors also describe locally refined algorithms for charge deposition and phase-space sampling, based on adaptive refinement trees. When mature, our Linearly-Transformed PIC (LTPIC) scheme will incorporate some of the multilevel refinement features presented in [16], and the resulting adaptive scheme should be compared with the CPK and $\mathrm{CM}$ methods to determine which classes of problems best fit each method.

The outline is as follows. In Section 2 we describe the LTPIC scheme for $1 \mathrm{~d} 1 \mathrm{v}$ electrostatic plasmas: in Section 2.1 we introduce the main notations and present the general form of the numerical solutions. Although a wide range of shape-functions is supported by our approach, to illustrate the method we review in Section 2.2 one deterministic algorithm for initialization and remapping of B-spline particles, and we provide a correction scheme to make the remappings conservative. In Section 2.3 we then define a particle transport scheme that transforms the shapes in phase space, and is solely based on pointwise evaluations of the (forward) numerical flow. Two specific charge deposition schemes are then presented in Section 2.4, and a leap-frog time advance scheme implementing the method is described in Section 2.5. Numerical results involving several standard benchmark tests and a halo problem associated with an initially mismatched thermal distribution are presented in Section 3 . The results obtained demonstrate some advantages of our method compared to the classical PIC or FSL approaches. They also indicate that for numerical choices involving similar computational effort, the LTPIC method is capable of accuracy comparable to or greater than state-of-the-art high-resolution Vlasov schemes. 


\section{The numerical method}

To describe our method we may consider the normalized 1d1v Vlasov-Poisson equation

$$
\left\{\partial_{t}+v \partial_{x}+E(x, t) \partial_{v}\right\} f(t, z)=0 \quad \text { with } t \geq 0, \quad z=(x, v) \in \mathbb{R}^{2},
$$

which models the evolution of simplified plasmas and sheet beams; see for instance Ref. [22]. Here, $x$ and $v$ are dimensionless positions and velocities and $E$ is a dimensionless electric field satisfying

$$
\partial_{x} E(t, x)=\int_{\mathbb{R}} f(t, x, v) \mathrm{d} v-n_{e},
$$

where $n_{e}$ is the density of a uniform neutralizing background cloud.

\subsection{Structure of the numerical solutions}

As in standard particle methods, we represent the phase-space density $f$ with weighted collections of finite-size particles (index $k$ ) which are pushed along their trajectories $z_{k}^{n}$ corresponding to the discrete times $t_{n}=n \Delta t, n=0,1 \ldots, N_{t}$. However, in our method the particles also have their shape transformed to better represent the local shear and rotation flows in phase space, as illustrated in Figure 1. The particles can either be structured or unstructured. The first case corresponds to the initialization and remapping steps, where particles are defined as tensor-product B-splines and centered on regular nodes

$$
z_{k}^{0}=\left(x_{k}^{0}, v_{k}^{0}\right) \equiv h k \quad \text { with } \quad k \in \mathbb{Z}^{2}=\{\ldots,-1,0,1, \ldots\}^{2} .
$$

Specifically, the univariate (i.e., one-dimensional) centered B-spline $\mathcal{B}_{p}$ is recursively defined as the piecewise polynomial of degree $p$ satisfying

$$
\mathcal{B}_{0}(x) \equiv\left\{\begin{array}{ll}
1, & -\frac{1}{2} \leq x \leq \frac{1}{2} \\
0 & \text { otherwise }
\end{array} \quad \text { and } \quad \mathcal{B}_{p}(x) \equiv \int_{x-\frac{1}{2}}^{x+\frac{1}{2}} \mathcal{B}_{p-1}(\tilde{x}) \mathrm{d} \tilde{x} \quad \text { for } \quad p \geq 1 .\right.
$$

Thus $\mathcal{B}_{1}(x)=\max \{1-|x|, 0\}$ is the traditional "hat-function", $\mathcal{B}_{3}$ is the well-known cubic Bspline supported on $[-2,2]$, and so on, see e.g. Ref. [23]. The fundamental shape function is then defined on the two-dimensional phase space as a tensor product

$$
\varphi(z) \equiv \mathcal{B}_{p}(x) \mathcal{B}_{p}(v) \quad \text { with support } \quad \operatorname{supp}(\varphi)=\left[-c_{p}, c_{p}\right]^{2}, \quad c_{p} \equiv \frac{p+1}{2},
$$

from which we derive a normalized $\left(\int \varphi_{h}=1\right)$, grid-scaled shape function $\varphi_{h}(z) \equiv h^{-2} \varphi\left(h^{-1} z\right)$. Structured particles are then defined as translated versions of the latter,

$$
\varphi_{h, k}^{0}(z) \equiv \varphi_{h}\left(z-z_{k}^{0}\right)=h^{-2} \varphi\left(h^{-1} z-k\right), \quad k \in \mathbb{Z}^{2} .
$$

When transported by our method, particles become unstructured in the sense that their centers $z_{k}^{n}$ leave the nodes of the structured phase-space grid and their shapes are linearly transformed. That is, the positions of different parts of the "cloud" associated with a single particle advance with their own peculiar velocities, the velocities advance with their own peculiar accelerations, and the cloud distorts, but the distortion is constrained to be linear. Generic particles are then 
characterized by the $2 \times 2$ deformation matrices $D_{k}^{n}$ (initialized with $\left.D_{k}^{0} \equiv\left(\begin{array}{ll}1 & 0 \\ 0 & 1\end{array}\right)\right)$ which determine the linear transformation of their shape, and numerical solutions take the form

$$
f_{h}^{n}(z)=\sum_{k \in \mathbb{Z}^{2}} w_{k}^{n} \varphi_{h, k}^{n}(z) \quad \text { with } \quad \varphi_{h, k}^{n}(z) \equiv \varphi_{h}\left(D_{k}^{n}\left(z-z_{k}^{n}\right)\right) .
$$

In Section 2.2 we shall describe a structured particle approximation operator

$$
A_{h}: f(z) \mapsto \sum_{k \in \mathbb{Z}^{2}} w_{k}(f) \varphi_{h}\left(z-z_{k}^{0}\right)
$$

acting on a generic density $f$, and in Section 2.3 and 2.5 we will construct a time-dependent transport operator

$$
T_{h}^{n}: \sum_{k \in \mathbb{Z}^{2}} w_{k}^{n} \varphi_{h}\left(D_{k}^{n}\left(z-z_{k}^{n}\right)\right) \mapsto \sum_{k \in \mathbb{Z}^{2}} w_{k}^{n+1} \varphi_{h}\left(D_{k}^{n+1}\left(z-z_{k}^{n+1}\right)\right) .
$$

The deformation matrices will be transformed with an area preserving scheme $\left(\operatorname{det}\left(D_{k}^{n+1}\right)=\right.$ $\operatorname{det}\left(D_{k}^{n}\right)=1$ ), so that the charges carried by the particles read (up to a constant factor)

$$
\int w_{k}^{n} \varphi_{h, k}^{n}(z) \mathrm{d} z=\int w_{k}^{n} \varphi_{h}\left(D_{k}^{n}\left(z-z_{k}^{n}\right)\right) \mathrm{d} z=w_{k}^{n} \int \varphi_{h}(\tilde{z}) \mathrm{d} \tilde{z}=w_{k}^{n}
$$

In particular, the particle weights will not be modified by our transport operator.

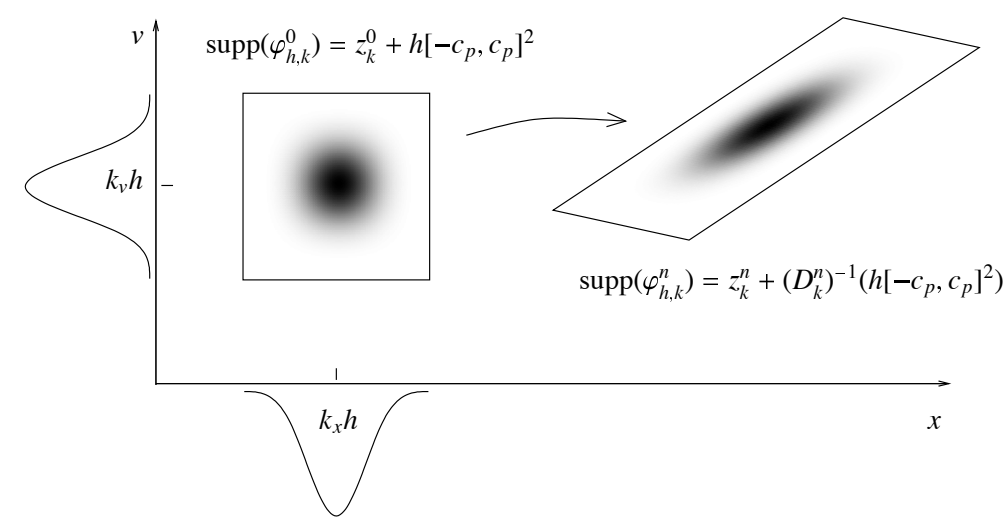

Figure 1: Structured particles (left) are defined at initialization and remapping steps, as tensor-product B-splines centered on regular nodes $z_{k}^{0}=h k, k \in \mathbb{Z}^{2}$. Unstructured particles (right) are obtained by pushing the particle centers along their trajectories $z_{k}^{n}$ and transforming their shapes with a matrix $D_{k}^{n}$ representing the local Jacobian of the characteristic flow.

To prevent the deformed particles from being arbitrarily stretched in one direction, we have chosen to periodically remap them onto the regular grid (3). Note that, as in Semi-Lagrangian methods, the remappings are likely to introduce unwanted numerical diffusion. However, since our particle method is mathematically proven to converge without remappings [16], we expect the optimal remapping frequencies to be significantly lower than with Semi-Lagrangian schemes. This point will be numerically demonstrated in Section 3 . 
The global structure of the scheme is then formulated as follows. First, a collection of weighted particles is initialized with

$$
f_{h}^{0} \equiv A_{h} f(t=0),
$$

then for $n=0, \ldots, N_{t}-1$, we compute

$$
f_{h}^{n+1} \equiv T_{h}^{n} f_{h}^{n, 0} \quad \text { where } \quad f_{h}^{n, 0} \equiv \begin{cases}A_{h} f_{h}^{n} & \text { if } n>0 \text { and } \bmod \left(n \Delta t, \Delta t_{\mathrm{r}}\right)=0, \\ f_{h}^{n} & \text { otherwise }\end{cases}
$$

to evolve the distribution with a given remapping period $\Delta t_{\mathrm{r}}$.

\subsection{Initialization and remappings}

Since the fundamental shape function $\varphi_{h}$ is a B-spline with the same scale as the spacing $h$ of the regular grid (3), arbitrary polynomials with coordinate degree less or equal to $p$ can be obtained by linear combinations of structured particles (6) derived by shifting $\varphi_{h}$ on the grid, see, e.g., [23]. Therefore, to initialize and remap the particle densities we can use existing high order approximation schemes that rely on that property. One attractive method is given by the quasiinterpolation schemes described in Refs. [24] and [25]. Such schemes pass through data points when they are described by polynomial target functions $f$ of a certain degree, and they have the advantage of computing high order B-spline approximants from local evaluations of the target function, unlike standard spline interpolation which requires solving a global system. Thus, in the univariate case the approximation $A_{h}^{(1 d)}$ takes the form

$$
A_{h}^{(1 d)}: f(x) \mapsto \sum_{k \in \mathbb{Z}} w_{k}(f) \varphi_{h}(x-h k) \quad \text { with weights } \quad w_{k}(f) \equiv h \sum_{|l| \leq m_{p}} a_{l} f(h(k+l)) .
$$

Here we have denoted $\varphi_{h}(x) \equiv h^{-1} \mathcal{B}_{p}\left(h^{-1} x\right)$, and the $a_{l}=a_{-l}$ are symmetric coefficients defined in such a way that $A_{h}^{(1 d)} f=f$ for any $f(x)=\alpha_{0}+\cdots+\alpha_{p} x^{p}$. Specifically, they can be computed with the algorithm from Ref. [24, Section 6]. For the first orders we find

- $m_{p}=0$ and $a_{0}=1$ for $p=1$,

- $m_{p}=1$ and $\left(a_{0}, a_{1}\right)=\left(\frac{8}{6},-\frac{1}{6}\right)$ for $p=3$,

- $m_{p}=4$ and $\left(a_{0}, a_{1}, a_{2}, a_{3}, a_{4}\right)=\left(\frac{503}{288},-\frac{1469}{3600}, \frac{7}{225}, \frac{13}{3600}, \frac{1}{14400}\right)$ for $p=5$.

In the bivariate case we can "tensorize" the above scheme, as it is easily checked that the operator

$$
A_{h}: f(z) \mapsto \sum_{k \in \mathbb{Z}^{2}} w_{k}(f) \varphi_{h}\left(z-z_{k}^{0}\right) \quad \text { with } \quad w_{k}(f) \equiv h^{2} \sum_{\|l\|_{\infty} \leq m_{p}} a_{l} f\left(z_{k+l}^{0}\right), \quad a_{l} \equiv a_{l_{x}} a_{l_{v}}
$$

reproducts any polynomial of coordinate degree less than or equal to $p$ (here, $\|l\|_{\infty} \equiv \max \left\{\left|l_{x}\right|,\left|l_{v}\right|\right\}$ ). With standard arguments one can then show that the resulting approximation error converges as $h^{p+1}$ for smooth functions $f$, see e.g., Ref. [16].

When the above scheme is used to remap a generic particle density $f_{h}^{n}$ of the form (7), the mass of the resulting approximation is (setting $a_{l} \equiv 0$ for $\|l\|_{\infty}>m_{p}$ and using $\sum_{l \in \mathbb{Z}^{2}} a_{l}=1$ )

$$
\int A_{h} f_{h}^{n}(z) \mathrm{d} z=\sum_{k \in \mathbb{Z}^{2}} w_{k}\left(f_{h}^{n}\right) \int \varphi_{h}\left(z-z_{k}^{0}\right) \mathrm{d} z=\sum_{k \in \mathbb{Z}^{2}} w_{k}\left(f_{h}^{n}\right)=h^{2} \sum_{k, l, k^{\prime} \in \mathbb{Z}^{2}} a_{l} w_{k^{\prime}}^{n} \varphi_{h, k^{\prime}}^{n}\left(z_{k+l}^{0}\right)=\sum_{k^{\prime} \in \mathbb{Z}^{2}} \hat{w}_{k^{\prime}}^{n}
$$


where $\hat{w}_{k^{\prime}}^{n}=h^{2} w_{k^{\prime}}^{n} \sum_{i \in \mathbb{Z}^{2}} \varphi_{h, k^{\prime}}^{n}\left(z_{i}^{0}\right)$ represents the charge deposited by the particle $k^{\prime}$ in the remapping process. Due to the shape transformations, this quantity generally differs from the original $w_{k^{\prime}}^{n}$ (indeed it vanishes if the support of $\varphi_{h, k^{\prime}}^{n}$ misses the grid $h \mathbb{Z}^{2}$ ). This shows that the quasiinterpolation is not conservative, but a locally conservative correction is easily implemented by depositing the local error $w_{k^{\prime}}^{n}-\hat{w}_{k^{\prime}}^{n}$ with a PIC-like method, which results in defining

$$
w_{k}^{n, 0}=w_{k}\left(f_{h}^{n}\right)+\sum_{k^{\prime} \in \mathbb{Z}^{2}}\left(w_{k^{\prime}}^{n}-\hat{w}_{k^{\prime}}^{n}\right) h^{2} \varphi_{h}\left(z_{k}^{0}-z_{k^{\prime}}^{n}\right) .
$$

Note that in practice the deposited fractions $\hat{w}_{k^{\prime}}^{n}$ can be evaluated by summing over $i \in \mathbb{Z}^{2}$ the values $\varphi_{h, k^{\prime}}^{n}\left(z_{i}^{0}\right)$ involved in the quasi-interpolation scheme.

\subsection{Particle transport with linear transformations}

The LTPIC scheme is based on a LTP (linearly-transformed particle) transport operator $T_{h}[\mathcal{F}]$ that transforms the particles through local linearizations of a given characteristic flow $\mathcal{F}$. In this section we present the LTP transport operator in this general setting, and reserve for the following sections the description of the numerical flow. Schematically, one could indeed decompose the transport operator $T_{h}^{n}$ appearing in (8) as follows.

1. From the density carried by the particles one computes a numerical flow

$$
f_{h}^{n} \mapsto \mathcal{F}_{h}^{n}
$$

that approximates the (exact) characteristic flow of the Vlasov equation (1) over one time step $\left[t_{n}, t_{n+1}\right]$. Namely, the mapping $\mathcal{F}_{\text {ex }}^{n}:(x, v) \mapsto(X, V)\left(t_{n+1}\right)$ that associates any phasespace point to the advanced-time point of its corresponding trajectory defined by

$$
\left\{\begin{array}{l}
X^{\prime}(t)=V(t) \\
V^{\prime}(t)=E(t, X(t))
\end{array} \quad \text { with initial data } \quad(X, V)\left(t_{n}\right)=(x, v) .\right.
$$

2. The particles are transported by the associated LTP transport operator,

$$
T_{h}^{n}=T_{h}\left[\mathcal{F}_{h}^{n}\right] .
$$

In Section 2.5 we will derive a leap-frog version of this approach, involving two intermediate flows $\mathcal{F}_{h}^{n, 0}$ and $\mathcal{F}_{h}^{n, 1}$ such that $\mathcal{F}_{h}^{n, 1} \circ \mathcal{F}_{h}^{n, 0} \approx \mathcal{F}_{\text {ex }}^{n}$. The LTP operator $T_{h}[\cdot]$ will then be applied twice per time step, i.e., we will define $T_{h}^{n} \equiv T_{h}\left[\mathcal{F}_{h}^{n, 1}\right] T_{h}\left[\mathcal{F}_{h}^{n, 0}\right]$. However, to simplify our presentation we shall consider in the remainder of this section that we are given a single flow $\mathcal{F}_{h}^{n} \approx \mathcal{F}_{\text {ex }}^{n}$.

Applied to a generic particle $\varphi_{h, k}^{n}$ with deformation matrix $D_{k}^{n}$, the LTP transport operator is

$$
T_{h}\left[\mathcal{F}_{h}^{n}\right]: \quad \varphi_{h, k}^{n} \equiv \varphi_{h}\left(D_{k}^{n}\left(\cdot-z_{k}^{n}\right)\right) \mapsto \varphi_{h, k}^{n+1} \equiv \varphi_{h}\left(D_{k}^{n+1}\left(\cdot-z_{k}^{n+1}\right)\right) \quad \text { with } \quad\left\{\begin{array}{l}
z_{k}^{n+1} \equiv \mathcal{F}_{h}^{n}\left(z_{k}^{n}\right) \\
D_{k}^{n+1} \equiv D_{k}^{n}\left(J_{k}^{n}\right)^{-1}
\end{array}\right.
$$

where $J_{k}^{n}$ is a matrix representing the Jacobian of the flow at $z_{k}^{n}$, defined as follows. An approximated Jacobian matrix $\tilde{J}_{k}^{n}$ is first defined with a centered finite difference scheme,

$$
\left(\tilde{J}_{k}^{n}\right)_{i, j} \equiv(2 h)^{-1}\left[\left(\mathcal{F}_{h}^{n}\right)_{i}\left(z_{k}^{n}+h \boldsymbol{e}_{j}\right)-\left(\mathcal{F}_{h}^{n}\right)_{i}\left(z_{k}^{n}-h \boldsymbol{e}_{j}\right)\right] \approx \partial_{j}\left(\mathcal{F}_{\mathrm{ex}}^{n}\right)_{i}\left(z_{k}^{n}\right) \quad \text { for } \quad 1 \leq i, j \leq 2,
$$


where we have denoted $\boldsymbol{e}_{j}=\left(\delta_{i, j}\right)_{1 \leq i \leq 2}$. Here $h$ is the grid spacing of the remapping grid, but a different spacing could be used as well. Next we observe that, while the exact flow has a Jacobian with uniform determinant equal to 1 , there is no reason why this should be true for the finite difference approximation (14). To obtain a conservative transport scheme (in the sense that $\left.\int \varphi_{h, k}^{n+1}(z) \mathrm{d} z=\int \varphi_{h, k}^{n}(z) \mathrm{d} z\right)$, we then define $J_{k}^{n}$ as

$$
J_{k}^{n} \equiv \operatorname{det}\left(\tilde{J}_{k}^{n}\right)^{-\frac{1}{2}} \tilde{J}_{k}^{n}
$$

To justify the above approximations inherent in Eqs. (13)-(15) we temporarily assume that we can apply the exact flow $\mathcal{F}_{\text {ex }}^{n}$. Pushing a fixed-shape particle as in standard PIC schemes gives

$$
T_{\mathrm{PIC}}\left[\mathcal{F}_{\mathrm{ex}}^{n}\right]: \varphi_{h}\left(z-z_{k}^{n}\right) \mapsto \varphi_{h}\left(z-z_{k}^{n+1}\right) \quad \text { with } \quad z_{k}^{n+1} \equiv \mathcal{F}_{\mathrm{ex}}^{n}\left(z_{k}^{n}\right) .
$$

Now, since (12) is reversible, the exact transport of an arbitrary phase-space density $f\left(t_{n}, z\right)$ over the time step is $f\left(t_{n+1}, z\right)=f\left(t_{n},\left(\mathcal{F}_{\mathrm{ex}}^{n}\right)^{-1}(z)\right)$. In particular, for the particle $\varphi_{h}\left(z-z_{k}^{n}\right)$ we have

$$
T_{\mathrm{ex}}\left[\mathcal{F}_{\mathrm{ex}}^{n}\right]: \varphi_{h}\left(z-z_{k}^{n}\right) \mapsto \varphi_{h}\left(\left(\mathcal{F}_{\mathrm{ex}}^{n}\right)^{-1}(z)-z_{k}^{n}\right),
$$

to which (16) can be seen as the lowest order approximation. Enhanced accuracy is obtained by a first order expansion around $z_{k}^{n}$ : writing $J_{\mathcal{F}}(z)=\left(\partial_{j} \mathcal{F}_{i}(z)\right)_{1 \leq i, j \leq 2}$ the Jacobian matrix associated with an arbitrary flow $\mathcal{F}: \mathbb{R}^{2} \rightarrow \mathbb{R}^{2}$, we let

$$
\mathcal{F}_{\mathrm{ex}, z_{k}^{n}}^{n}(z) \equiv \mathcal{F}_{\mathrm{ex}}^{n}\left(z_{k}^{n}\right)+J_{\mathcal{F}_{\mathrm{ex}}^{n}}\left(z_{k}^{n}\right)\left(z-z_{k}^{n}\right)
$$

denote the linearized flow around $z_{k}^{n}$. We then define a "formal" LTP transport operator as the exact tranport corresponding to this linearized flow, namely

$$
T_{\mathrm{LTP}}\left[\mathcal{F}_{\mathrm{ex}}^{n}\right] \equiv T_{\text {ex }}\left[\mathcal{F}_{\text {ex }, z_{k}^{n}}^{n}\right] \quad \text { for the particle associated to } z_{k}^{n} .
$$

Applied to a structured particle, we observe that it reads

$$
T_{\mathrm{LTP}}\left[\mathcal{F}_{\mathrm{ex}}^{n}\right]: \varphi_{h}\left(z-z_{k}^{n}\right) \mapsto \varphi_{h}\left(\left(J_{k}^{n}\right)^{-1}\left(z-z_{k}^{n+1}\right)\right) \quad \text { with } \quad z_{k}^{n+1} \equiv \mathcal{F}_{\mathrm{ex}}^{n}\left(z_{k}^{n}\right), \quad J_{k}^{n} \equiv J_{\mathcal{F}_{\mathrm{ex}}^{n}}\left(z_{k}^{n}\right) .
$$

Now, replacing the flow $\mathcal{F}_{\text {ex }}^{n}$ by its numerical approximation $\mathcal{F}_{h}^{n}$ and using a finite difference scheme for the forward Jacobian leads then to the practical LTP transport operator as defined by Eqs. (13)-(15). A rigorous error analysis of this procedure is presented in [16]. This error analysis demonstrates the global convergence of both the discrete (13) and continuous (18) schemes (without remappings) in the uniform norm, as $h$ tends to 0 , provided that the exact flow is approximated with sufficient accuracy.

\subsection{Conservative charge deposition schemes for linearly transformed particles}

To complete the LTPIC scheme we now describe how to compute the field from the linearly transported particles. For this purpose we equip the (1d) physical space with regular nodes

$$
x_{i}=i h^{\prime} \quad \text { with } \quad i \in \mathbb{Z},
$$

and as in Section2.2 we let $\varphi_{h^{\prime}}(x) \equiv \frac{1}{h^{\prime}} \mathcal{B}_{p}\left(\frac{x}{h^{\prime}}\right)$ denote the scaled B-spline of mass 1 in the physical variable. Following the standard approach [26] we represent the charge density on the grid with

$$
\rho_{h^{\prime}}^{n}(x) \equiv \sum_{i \in \mathbb{Z}} \rho_{i}^{n} \varphi_{h^{\prime}}\left(x-x_{i}\right),
$$


and solve the Poisson equation on the same grid. Specifically we represent the electric field with

$$
E_{h^{\prime}}^{n}(x) \equiv \sum_{i \in \mathbb{Z}} h^{\prime} E_{i}^{n} \varphi_{h^{\prime}}\left(x-x_{i}\right)
$$

with coefficients computed by a centered finite difference scheme, such as

$$
E_{i}^{n}=-\frac{\phi_{i+1}^{n}-\phi_{i-1}}{2 h^{\prime}}, \quad-\frac{\phi_{i+1}^{n}-2 \phi_{i}+\phi_{i-1}}{\left(h^{\prime}\right)^{2}}=\rho_{h}^{n}\left(x_{i}\right) .
$$

Here the different normalization in (19) and (20) allow the coefficients $E_{i}^{n}$ to be on the order of the point values $E_{h^{\prime}}^{n}\left(x_{i}\right)$, whereas the coefficients $\rho_{i}^{n}$ are on the order of the local charges $h^{\prime} \rho_{h^{\prime}}^{n}\left(x_{i}\right)$, consistent with standard notations, see [26, Chapter 5]. To compute the local charges $\rho_{i}^{n}$, several methods can be considered.

1. In the simplest approach the particles are seen as point particles and they deposit their charges in a way similar to PIC schemes, i.e.,

$$
\rho_{i}^{n} \equiv h^{\prime} \sum_{k \in \mathbb{Z}^{2}} w_{k}^{n} \varphi_{h^{\prime}}\left(x_{k}^{n}-x_{i}\right) .
$$

Note that in this case the numerical scheme still differs from a standard PIC method, because the particles are periodically remapped on the regular grid with a smoothing effect.

2. To take into account the shape of the particles, specific deposition schemes can be used instead. They rely on an intermediate charge density defined as the exact integral of $f_{h}^{n}$ along the velocity variable,

$$
\tilde{\rho}_{h}^{n}(x)=\sum_{k \in \mathbb{Z}^{2}} \tilde{\rho}_{h, k}^{n}(x) \equiv \sum_{k \in \mathbb{Z}^{2}} w_{k}^{n} \int_{\mathbb{R}} \varphi_{h, k}^{n}(x, v) \mathrm{d} v=\int_{\mathbb{R}} f_{h}^{n}(x, v) \mathrm{d} v,
$$

and on the use of univariate quasi-interpolation (9) to compute the local charges,

$$
\rho_{h^{\prime}}^{n} \equiv A_{h^{\prime}}^{(1 d)} \tilde{\rho}_{h}^{n} \quad \text { i.e., } \quad \rho_{i}^{n} \equiv h^{\prime} \sum_{|l| \leq m_{p}} a_{l} \tilde{\rho}_{h}^{n}\left(x_{i+l}\right)=h^{\prime} \sum_{k \in \mathbb{Z}^{2}} \sum_{|l| \leq m_{p}} a_{l} \tilde{\rho}_{h, k}^{n}\left(x_{i+l}\right) .
$$

Note that a correction similar to (11) can be used here to make the deposition conservative, in the sense that $\int \rho_{h}^{n}(x) \mathrm{d} x=\int f_{h}^{n}(z) \mathrm{d} z$. In the above deposition formula (22), we observe that the evaluation of the "integrated particles" $\tilde{\rho}_{h, k}^{n}$ is not straightforward, due to the linear transformation of their shape. To compute them we have considered two methods.

2.a. In the first method (see Algorithm 2.1 and Figure 2) we use a Gaussian quadrature $\hat{\rho}_{h, k}^{n}\left(x_{i+l}\right)$ to evaluate each velocity integral $\tilde{\rho}_{h, k}^{n}\left(x_{i+l}\right)$. In order to be accurate this approximation requires a few quadrature intervals fitted to the particle support (projected along the velocity variable) and a few Gauss points per interval. This makes it hard to apply in higher dimensions.

2.b. In the second method (see Algorithm 2.2 and Figure 3) we simply replace each integrated particle by a univariate weighted B-spline sharing the same first 3 moments. Specifically, we approximate $\tilde{\rho}_{h, k}^{n}$ as

$$
\tilde{\rho}_{h, k}^{n}(x) \approx \hat{\rho}_{h, k}^{n}(x) \equiv \frac{w_{h, k}^{n}}{\lambda_{h, k}^{n}} \mathcal{B}_{p}\left(\frac{x-x_{k}^{n}}{\lambda_{h, k}^{n}}\right) \quad \text { with } \quad \lambda_{h, k}^{n} \equiv h \sqrt{\left(\left(D_{k}^{n}\right)_{2,2}\right)^{2}+\left(\left(D_{k}^{n}\right)_{1,2}\right)^{2}},
$$

so that $\int_{\mathbb{R}} x^{m} \hat{\rho}_{h, k}^{n}(x) \mathrm{d} x=\int_{\mathbb{R}} x^{m} \tilde{\rho}_{h, k}^{n}(x) \mathrm{d} x$ holds for $m=0,1,2$. The resulting implementation is much simpler, and the extension to higher dimensions is straightforward. 
Algorithm 2.1 (Charge deposition with Gaussian quadrature). Let $N_{G}$ and $N_{G}^{\prime}$ denote the prescribed number of quadrature intervals and Gauss points per interval in the $v$ dimension, per particle.

1. Loop over every active particle $\varphi_{h, k}^{n}$, i.e., over $k \in \mathbb{Z}^{2}$ such that $w_{k}^{n} \neq 0$. For conciseness we denote the deformation matrix by $D \equiv D_{k}^{n}$, and we observe that the particle support is

$$
\operatorname{supp}\left(\varphi_{h, k}^{n}\right)=\left(x_{k}^{n}, v_{k}^{n}\right)+D^{-1}\left(h\left[-c_{p}, c_{p}\right]^{2}\right)=\left\{(x, v):\left\|D\left(x-x_{k}^{n}, v-v_{k}^{n}\right)\right\|_{\infty} \leq h c_{p}\right\} .
$$

2. Determine the $x$-projection $\left[x_{k}^{n}-h_{x}, x_{k}^{n}+h_{x}\right]$ of the support (24) of $\varphi_{h, k}^{n}$, i.e., set

$$
h_{x}=h_{x}(h, k, n) \equiv \frac{1}{2} \operatorname{diam}\left(\left\{x_{k}^{n}+\left(D^{-1} r\right)_{x}:\|r\|_{\infty} \leq h c_{p}\right\}\right)=h c_{p}\left(\left|D_{2,2}\right|+\left|D_{1,2}\right|\right),
$$

where we have used $D^{-1}=\left(\begin{array}{cc}D_{2,2} & -D_{1,2} \\ -D_{2,1} & D_{1,1}\end{array}\right)$ since $\operatorname{det}(D)=1$.

3. Loop over the non-vanishing point values $\tilde{\rho}_{h, k}^{n}\left(x_{l^{\prime}}\right)$, namely over $l^{\prime} \in \mathbb{Z}$ such that

$$
\left|x_{k}^{n}-x_{l^{\prime}}\right|<h_{x} \text {, i.e., } l^{\prime} \in\left\{\left\lfloor\frac{1}{h^{\prime}}\left(x_{k}^{n}-h_{x}\right)\right\rfloor+1, \cdots,\left[\frac{1}{h^{\prime}}\left(x_{k}^{n}+h_{x}\right)\right]-1\right\} .
$$

Then define $\hat{\rho}_{h, k}^{n}\left(x_{l^{\prime}}\right) \approx \tilde{\rho}_{h, k}^{n}\left(x_{l^{\prime}}\right)$ with $N_{G}$ Gauss quadrature formulas using $N_{G}^{\prime}$ points in the $v$ dimension: from (24) the interval $\left[v^{-}\left(l^{\prime}\right), v^{+}\left(l^{\prime}\right)\right] \equiv \operatorname{supp}\left(v \mapsto \varphi_{h, k}^{n}\left(x^{\prime}, v\right)\right)$ is given by

$$
\left\{\begin{array}{l}
v^{-}\left(l^{\prime}\right) \equiv v_{k}^{n}+\max _{i=1,2}\left\{\left(D_{i, 2}\right)^{-1}\left(D_{i, 1}\left(x_{k}^{n}-x_{l^{\prime}}\right)-h c_{p}\right)\right\} \\
v^{+}\left(l^{\prime}\right) \equiv v_{k}^{n}+\min _{i=1,2}\left\{\left(D_{i, 2}\right)^{-1}\left(D_{i, 1}\left(x_{k}^{n}-x_{l^{\prime}}\right)+h c_{p}\right)\right\}
\end{array}\right.
$$

so that we may compute

$$
\begin{aligned}
\hat{\rho}_{h, k}^{n}\left(x_{l^{\prime}}\right) & \equiv w_{k}^{n} \Delta v \sum_{m^{\prime}=0}^{N_{G}-1} \sum_{m=1}^{N_{G}^{\prime}} \lambda_{m}^{G} \varphi_{h, k}^{n}\left(x_{l^{\prime}}, v^{-}\left(l^{\prime}\right)+\left(m^{\prime}+v_{m}^{G}\right) \Delta v\right) \\
& \approx w_{k}^{n} \int_{v^{-}\left(l^{\prime}\right)}^{v^{+}\left(l^{\prime}\right)} \varphi_{h, k}^{n}\left(x_{l^{\prime}}, v\right) \mathrm{d} v=\tilde{\rho}_{h, k}^{n}\left(x_{l^{\prime}}\right) .
\end{aligned}
$$

Here, $\Delta v \equiv\left(v^{+}\left(l^{\prime}\right)-v^{-}\left(l^{\prime}\right)\right) / N_{G}$, and $\lambda_{i}^{G}, v_{i}^{G}$ are the Gauss weights and nodes corresponding to the interval $[0,1]$, e.g.,

$$
\begin{aligned}
& \text { for } N_{G}^{\prime}=1: \quad v_{1}^{G}=\frac{1}{2}, \quad \lambda_{1}^{G}=1, \\
& \text { for } N_{G}^{\prime}=2: \quad v_{i}^{G}=\frac{1}{2}\left(1 \pm \frac{1}{\sqrt{3}}\right), \quad \lambda_{i}^{G}=\frac{1}{2}, \quad i=1,2, \\
& \text { for } N_{G}^{\prime}=3:\left\{\begin{array}{l}
v_{2}^{G}=\frac{1}{2}, \quad \lambda_{2}^{G}=\frac{4}{9} \\
v_{i}^{G}=\frac{1}{2}\left(1 \pm \frac{\sqrt{15}}{5}\right), \quad \lambda_{i}^{G}=\frac{5}{18}, \quad i=1,3 .
\end{array}\right.
\end{aligned}
$$

Finally update the appropriate weights (initialized to 0 ) consistent with (22), by setting

$$
\rho_{i}^{n} \equiv \rho_{i}^{n}+h^{\prime} a_{l^{\prime}-i} \hat{\rho}_{h, k}^{n}\left(x_{l^{\prime}}\right) \quad \text { for } \quad i=l^{\prime}-l=l^{\prime}-m_{p}, \ldots, l^{\prime}+m_{p} .
$$

Algorithm 2.2 (Charge deposition with a moment method).

1. Loop over the active particles $\varphi_{h, k}^{n}$, i.e., over $k \in \mathbb{Z}^{2}$ such that $w_{k}^{n} \neq 0$. 


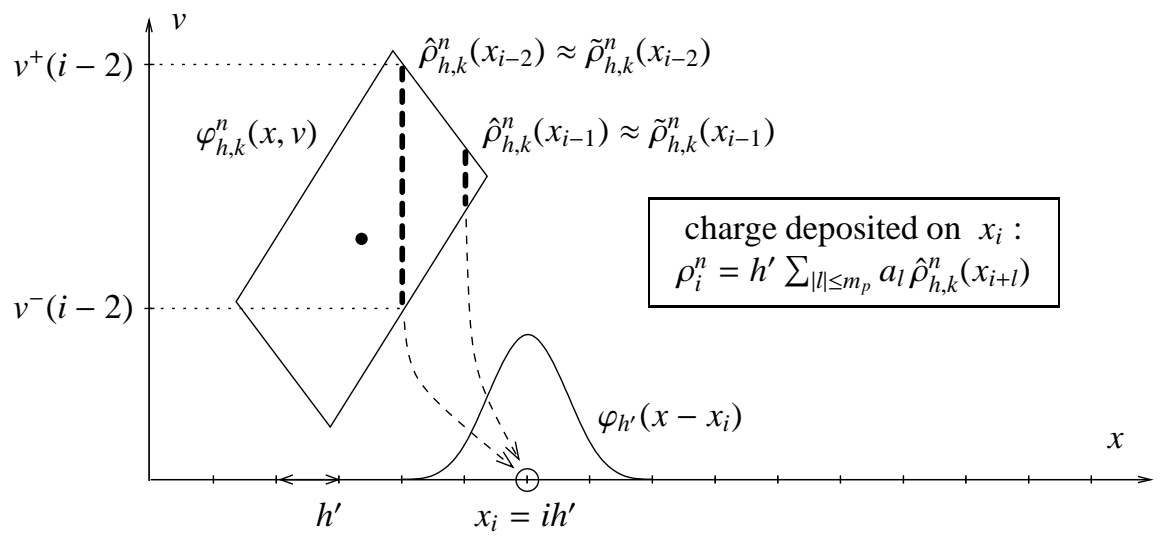

Figure 2: In the Gauss deposition scheme described in Algorithm 2.1 the charge density $\tilde{\rho}_{h, k}^{n}(x) \equiv w_{k}^{n} \int_{\mathbb{R}} \varphi_{h, k}^{n}(x, v) \mathrm{d} v$ associated to a linearly-transformed particle is deposited with a quasi-interpolation scheme where the required point values are estimated with Gaussian quadrature along $v$ slices.

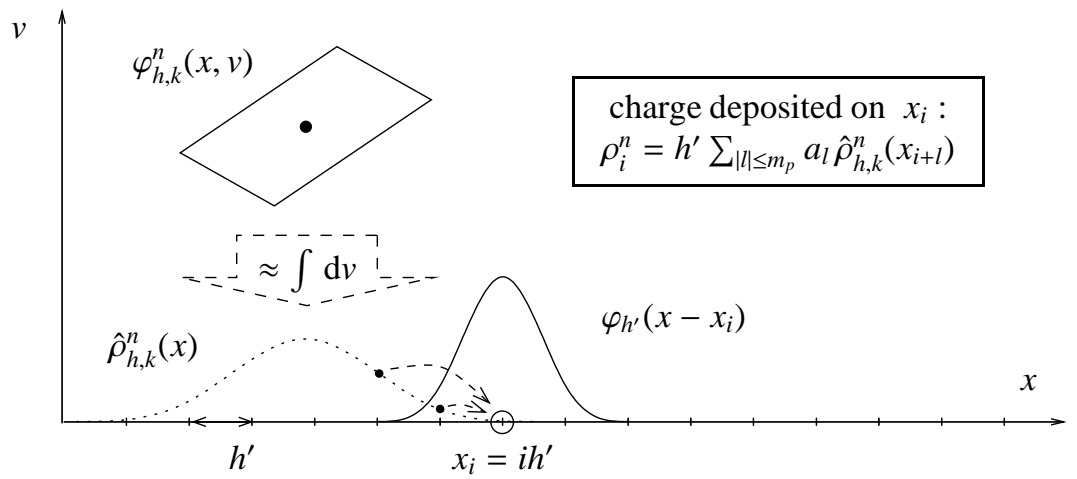

Figure 3: In the moment deposition scheme described in Algorithm 2.2 no numerical integration is needed. Instead, the charge density $\tilde{\rho}_{h, k}^{n}(x) \equiv w_{k}^{n} \int_{\mathbb{R}} \varphi_{h, k}^{n}(x, v) \mathrm{d} v$ is replaced by a B-spline $\hat{\rho}_{h, k}^{n}(x)$ that shares its first 3 moments, and the latter is deposited with the quasi-interpolation scheme (compare with Figure 2 .

2. Determine the support $\left[x_{k}^{n}-h_{x}, x_{k}^{n}+h_{x}\right]$ of $\hat{\rho}_{h, k}^{n}$, i.e., set $h_{x}=h_{x}(h, k, n) \equiv \lambda_{h, k}^{n} c_{p}$ with $\lambda_{h, k}^{n}$ defined as in (23).

3. Deposit the approximated charge contributions as above: loop over $l^{\prime} \in \mathbb{Z}$ satisfying (26), and update the weights as in (28), with the explicit expression (23) for $\hat{\rho}_{h, k}^{n}$.

The good news is that in practice it does not seem necessary to resort to accurate piecewise Gauss quadratures. Indeed, in most of the numerical tests presented in Section 3 the results obtained with the simple moment deposition scheme (displayed) were compared with simulations using a Gauss deposition scheme with $N_{G}=4$ quadrature intervals and $N_{G}^{\prime}=3$ Gauss points per intervals, and the differences were hardly visible. Maybe more surprisingly, we also com- 
pared these results with simulations using the much simpler, PIC-like point deposition scheme, and again the differences were hardly visible. This suggests that in many cases the oscillatory representation of the density (as seen by the field solver) still yields an electric field that is able to drive accurate dynamics. On one hand, we should not be overly surprised by such a fact, indeed it is routinely observed in PIC (and FSL) simulations. On the other hand, we could expect that in some cases an accurate resolution of the field requires a smooth representation of the density as seen by the solver, especially when high order solvers are used. This is an important question that is familiar in the finite element community, and that shall be addressed in future research.

\subsection{Time marching scheme}

Equipped with the LTP transport operator $T_{h}[\mathcal{F}]$ defined in (13) for a generic flow $\mathcal{F}$, and with the field solver described in Section 2.4, we are now in position to specify the numerical transport involved in the scheme (8), namely the operator

$$
T_{h}^{n}: f_{h}^{n, 0} \mapsto f_{h}^{n+1}
$$

To this end we consider a standard leap-frog time discretization. We first transport

$$
f_{h}^{n, 1} \equiv T_{h}\left[\mathcal{F}_{h}^{n, 0}\right] f_{h}^{n, 0} \quad \text { where } \quad \mathcal{F}_{h}^{n, 0}(x, v) \equiv\left(x+\frac{\Delta t}{2} v, v\right),
$$

then compute an intermediate electric field

$$
f_{h}^{n, 1} \mapsto E_{h^{\prime}}^{n, 1}
$$

using finite differences as described in Section 2.4 and finally complete the time step with

$$
f_{h}^{n+1} \equiv T_{h}\left[\mathcal{F}_{h}^{n, 1}\right] f_{h}^{n, 1} \quad \text { where } \quad \mathcal{F}_{h}^{n, 1}(x, v) \equiv\left(x+\frac{\Delta t}{2} \tilde{v}, \tilde{v} \equiv v+\Delta t E_{h^{\prime}}^{n, 1}(x)\right) .
$$

\section{Numerical simulations}

In this section we apply our LTPIC scheme (8) on a series of $1 \mathrm{~d} 1 \mathrm{v}$ test cases and compare the resulting solutions with classical PIC or FSL runs, the latter being obtained by freezing the particle shapes in our code - that is, by setting $D_{k}^{n} \equiv\left(\begin{array}{ll}1 & 0 \\ 0 & 1\end{array}\right)$ for all $k$ and $n$. To facilitate the comparison with LTPIC and FSL, we often indicate the number of particles used in a PIC run as a product (e.g., $128 \times 128$ ). This may correspond to a uniform grid where (weighted) particles are initialized, but in most cases the displayed PIC runs use unweighted particles initialized with a standard quiet start method.

In Sections 3.1 and 3.2 we first consider standard benchmark problems for which our results can be compared to the existing literature, see e.g. Refs. [27, 28, 29, 30, 31, 32]. Next, in Section 3.3 we study a more applied test case consisting of a mismatched beam in a constant (continous) focusing channel, derived from the 1d sheet beam model developed in Ref. [22].

For the benchmark test cases in Sections 3.1 and 3.2 we classically consider the normalized Vlasov equation (1) in $x$-periodic phase space $[0, L] \times \mathbb{R}$ coupled with a periodic Poisson equation

$$
\partial_{x} E(t, x)=\int_{\mathbb{R}} f(t, x, v) \mathrm{d} v-n_{e}, \quad t>0, \quad x \in[0, L] .
$$

Here $n_{e}$ is the uniform (and constant) density of a neutralizing background cloud, and we complete (29) with the standard condition $\int_{0}^{L} E(t, x) \mathrm{d} x=0$. 


\subsection{Weak and Strong Landau damping}

We first consider the normalized Vlasov-Poisson system described above with perturbed initial distribution

$$
f(t=0, x, v) \equiv \frac{1}{\sqrt{2 \pi}} \exp \left(-\frac{v^{2}}{2}\right)(1+A \cos (k x)) .
$$

Consistent with classical benchmarks [27, 28, 32] we take $k \equiv 0.5$ and set the perturbation amplitude $A \equiv 0.01$ for the weak Landau damping test case, or $A \equiv 0.5$ for the strong Landau damping test case (actually, for such a perturbation the field is only damped for times $t \lesssim 10$ ). In this section we use a cutoff velocity $v_{\max } \equiv 6.5$ and periodic boundary conditions at $x=0$ and $x=L \equiv 2 \pi / k$.

In Figure 4 we show the $L^{2}$ norms of the electric field (left panel, semi-log scale) and of the phase-space density (right panel) obtained with PIC and LTPIC simulations of the weak Landau damping. Results clearly show the noiseless aspect of the LTPIC method, as the theoretical damping rate $(\gamma=-0.1533)$ is matched with a low-resolution run using 64 Poisson cells and 64 particles per cell. We also observe the classical recurrent relaxation occuring with period $T_{R} \approx 60$, in good agreement with the theoretical period $L / \Delta v \approx 62$, see e.g., Ref. [27]. In contrast, a PIC run using the same number of cells and particles (labelled as PIC $_{1}$ ) is unable to predict the correct damping rate beyond $t \approx 5$. And even with 1024 particles per cell (and significantly greater cpu time), the $\mathrm{PIC}_{3}$ run only predicts the correct rate until $t=20$. We also see that the low-resolution LTPIC run does a significantly better job at preserving the $L^{2}$ norm of the density (a Vlasov invariant), compared to the low- and moderate-resolution PIC runs. For such a test case, only the high-resolution PIC run performs better with regard to the $L^{2}$ measure.

In Figure 5 the same quantities are shown for the strong Landau "damping". Again, the low-resolution LTPIC run predicts the benchmarked rates for the initial damping and subsequent exponential growth. The low-resolution $\mathrm{PIC}_{1}$ run (with 64 cells and $64 \times 64$ particles) only predicts the initial damping. The moderate-resolution $\mathrm{PIC}_{2}$ run predicts correctly both rates (although with less accuracy for the growth period), but at a significantly higher cost in terms of memory and cpu time. As for the preservation of the $L^{2}$ norm of the density we observe that LTPIC does not perform better than PIC here, essentially due to the remappings.

Finally, we found that the low-resolution FSL runs (using 64 cells and $64 \times 64$ particles) give energy curves very similar to the LTPIC ones, in both the weak and strong damping cases. These curves were omitted for readability.

To better assess the noiseless aspect of our method, we also show in Figure 6 the phase-space density $f_{h}^{n}(x, v)$ as it evolves in the time range $t_{n} \in[0,60]$, obtained with an LTPIC run with periodic $\left(\Delta t_{\mathrm{r}}=4\right)$ remappings on a grid of $256 \times 256$ particles. Here the strong phase-space filamentation is accurately resolved. In particular it agrees very well with similar phase-space plots shown on Figure 10 in Ref. [32], obtained with a high order Backward Semi-Lagrangian Discontinuous Galerkin (BSL-DG) scheme (our color scale is chosen in order to fit theirs). In that scheme the phase-space density is computed using a cartesian mesh of lower resolution in the $x$ dimension (128 points), but in the $v$ dimension where the filaments are most difficult to resolve the resolution is the same ( 256 points). Since each fifth-order DG cell contains 15 basis functions, we observe that this run involves about the same number of degrees of freedom as the LTPIC run, where each particle carries 6 floating numbers (for the weight, phase space coordinates and normalized deformation matrix). Close examination reveals slightly better resolution of fine structures with the BSL-DG scheme. However, due to the CFL constraint we note that in the BSL run the time steps are significantly smaller (namely $\Delta t \approx 0.03$ ) than with the present 
$L_{2}\left(E_{h^{\prime}}^{n}\right)$

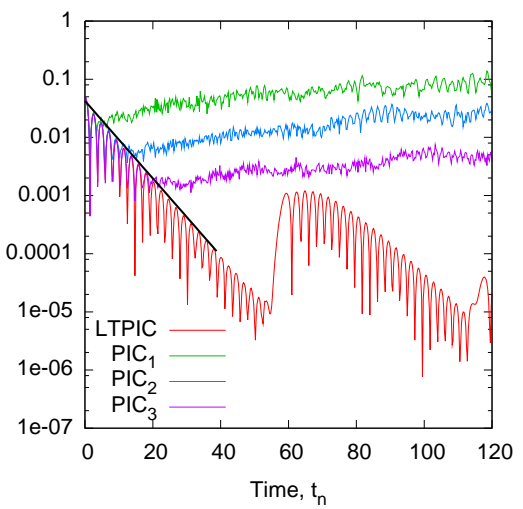

$L_{2}\left(f_{h}^{n}\right)-L_{2}\left(f_{h}^{0}\right)$

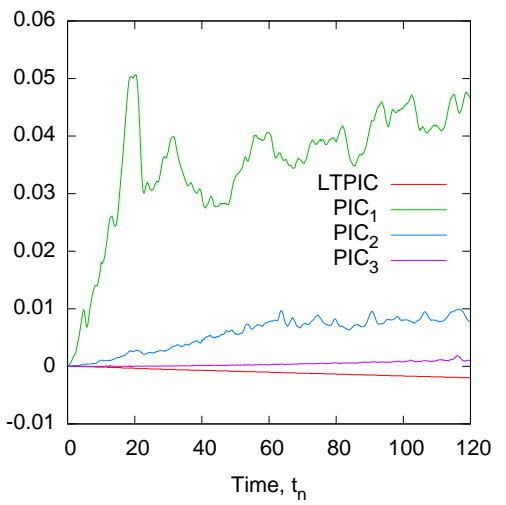

Figure 4: Weak Landau damping. $L^{2}$ norms of the electric field $E_{h^{\prime}}^{n}$ (left) and of the particle density $f_{h}^{n}$ (right) are plotted vs. $t_{n}=n \Delta t$ for LTPIC and PIC simulations. All the runs use a time step of $\Delta t=1 / 8$ and 64 cells for the Poisson solver. The PIC runs labelled as $\mathrm{PIC}_{1}, \mathrm{PIC}_{2}$ and $\mathrm{PIC}_{3}$ use increasingly high numbers of particles, namely $64 \times 64,128 \times 128$ and $256 \times 256$. The LTPIC run uses $64 \times 64$ particles and a remapping period $\Delta t_{\mathrm{r}}=4$. The approximate cpu times for these runs are $40 \mathrm{~s}\left(\mathrm{PIC}_{1}\right), 90 \mathrm{~s}\left(\mathrm{PIC}_{2}\right), 330 \mathrm{~s}\left(\mathrm{PIC}_{3}\right)$ and $45 \mathrm{~s}$ (LTPIC). On the left panel the plotted slope $(\gamma=-0.1533)$ matches the theoretical damping rate, see Refs. [28, 32]. The quasi-periodic relaxation in the LTPIC curve is known as a Poincaré recurrence, see text for details.
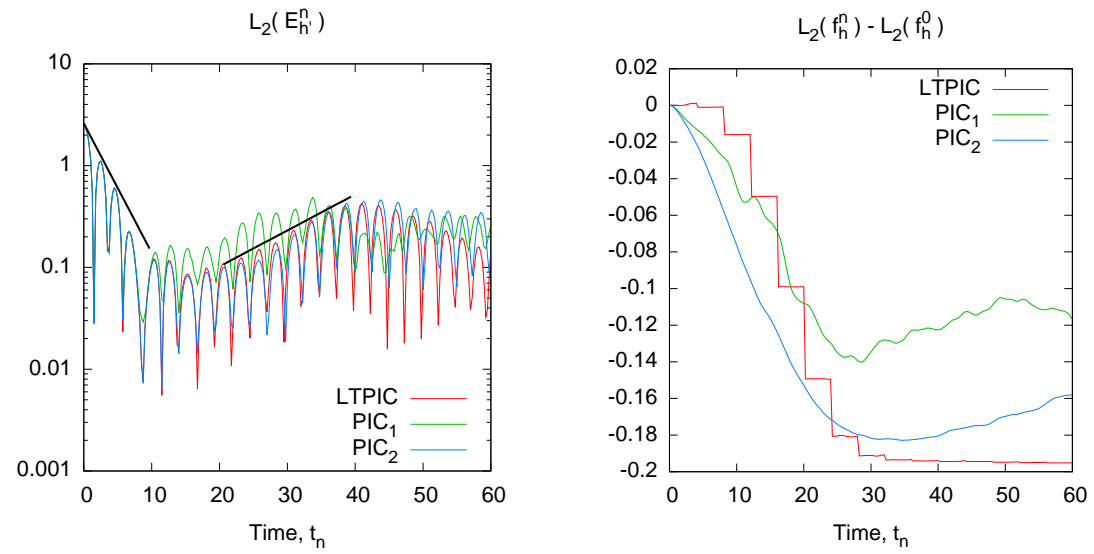

Figure 5: Strong Landau damping. Plotted quantities and numerical parameters for the PIC and LTPIC runs are the same than in Figure 4 The approximate cpu times for these runs are $20 \mathrm{~s}\left(\mathrm{PIC}_{1}\right), 45 \mathrm{~s}\left(\mathrm{PIC}_{2}\right)$ and $24 \mathrm{~s}$ (LTPIC). The plotted slopes $\left(\gamma_{1}=-0.2920\right.$ for the initial damping and $\gamma_{2}=0.0815$ for the growth between times $t=20$ and $\left.t=40\right)$ match benchmarked exponential rates, see e.g. Refs. [28, 32]. 
scheme where we have set $\Delta t=1 / 8$. Comparison with another fifth-order BSL-DG simulation [31, Fig. 7, bottom row] shows a better resolution for the LTPIC scheme, however in this case the BSL-DG run uses far less degrees of freedom than the LTPIC one.

$f_{h}^{n}(x, v)$ at $t_{n}=0$

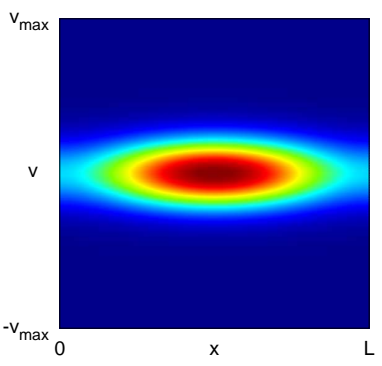

$f_{h}^{n}(x, v)$ at $t_{n}=18$

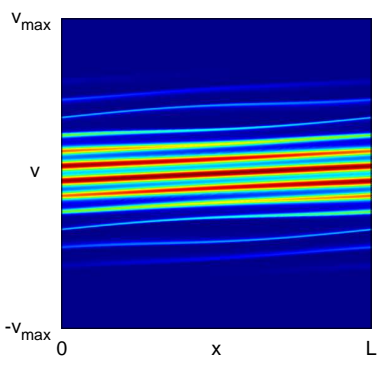

$f_{h}^{n}(x, v)$ at $t_{n}=45$

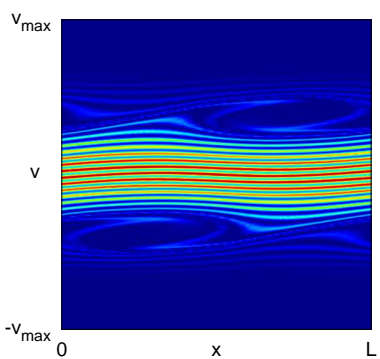

$f_{h}^{n}(x, v)$ at $t_{n}=6$

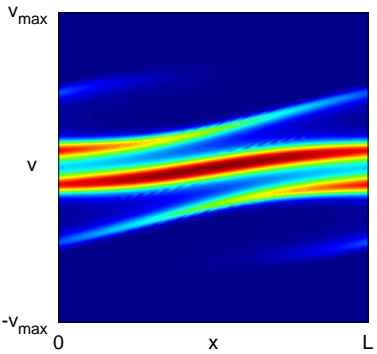

$f_{h}^{n}(x, v)$ at $t_{n}=27$

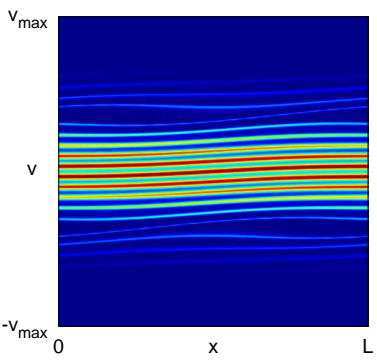

$f_{h}^{n}(x, v)$ at $t_{n}=54$

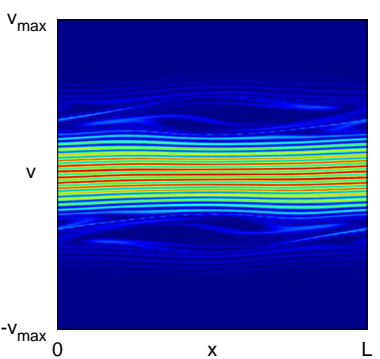

$f_{h}^{n}(x, v)$ at $t_{n}=9$

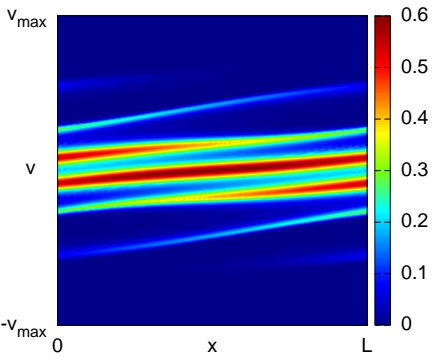

$f_{h}^{n}(x, v)$ at $t_{n}=36$

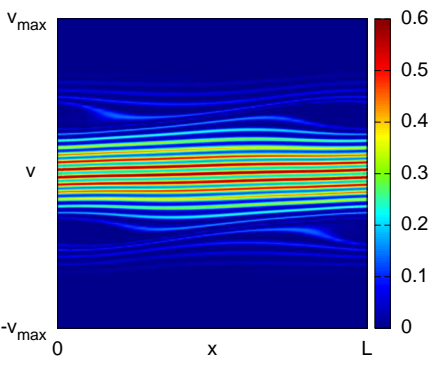

$f_{h}^{n}(x, v)$ at $t_{n}=60$

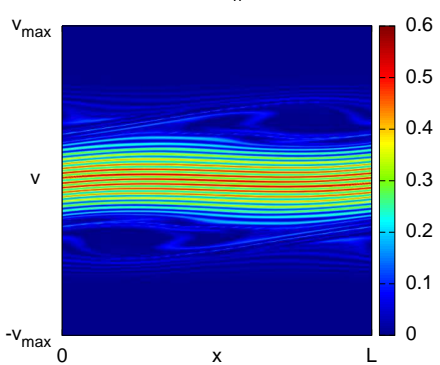

Figure 6: Strong Landau damping. Time evolution of the phase-space particle density $f_{h}^{n}(x, v)$ obtained with an LTPIC simulation. This run uses a time step $\Delta t=1 / 8$, a remapping period $\Delta t_{\mathrm{r}}=4$, a Poisson solver with 256 cells and $256 \times 256$ particles. On the $t_{n}=6$ snapshot some oscillations are visible but they almost vanish in the subsequent plots, due to the particle remappings and the strong shearing of the flow. The approximate cpu times for this runs is $660 \mathrm{~s}$.

In Figures 7 and 8 we then compare how well different particle methods (namely PIC, LTPIC and FSL with various numerical parameters indicated in the figure caption) resolve the filaments in the strong Landau "damping" test case at $t=60$. Phase-space densities obtained at $t=60$ with different schemes are shown on Figure 7 together with a reference solution obtained with an LTPIC run using improved numerical parameters relative to Figure 6. Again, the results clearly show that LTPIC and FSL are able to remove the noise. With PIC the localization of global patterns such as filaments and holes may be accurate, however the noise level is significant (and it remains so with finer simulations, not shown here). Results also show the effect of varying 
the remapping period $\Delta t_{\mathrm{r}}$ in the FSL and LTPIC runs. For low remapping periods both methods give similar results, which is expected since particles do not have time to deform much. For high remapping periods however the LTPIC performs significantly better: it introduces less diffusion than FSL, and does not present the unphysical oscillations that start to appear in the filaments computed with the FSL method. This is also expected from the convergence analysis of the LTP transport operator (17), which does not require remappings for asymptotic convergence. The good news is that this improved performance does not come at an expensive price: the measured cpu times are indeed similar for FSL and LTPIC runs, which indicates that the additional work of updating the deformation matrices does not represent a significant portion of the overall time.

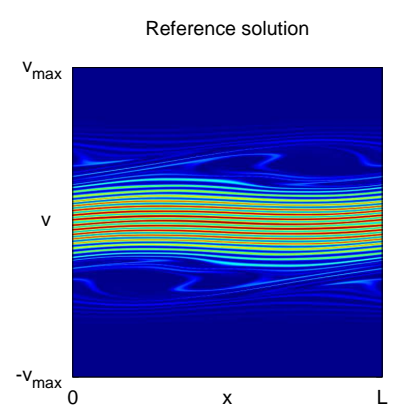

FSL with $\Delta \mathrm{t}_{\mathrm{r}}=1$

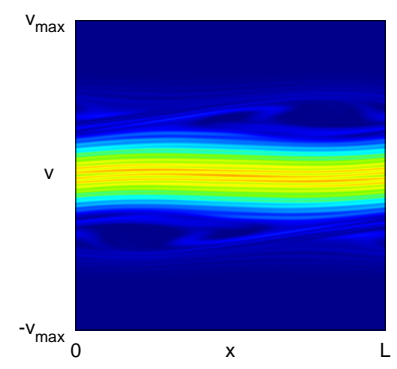

LTPIC with $\Delta \mathrm{t}_{\mathrm{r}}=1$

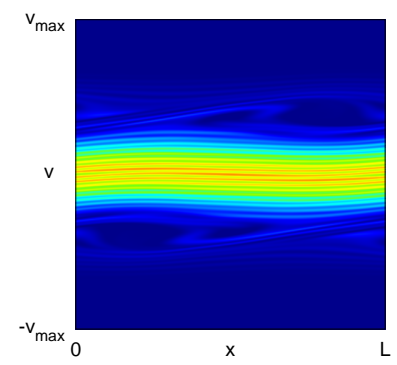

unweighted PIC

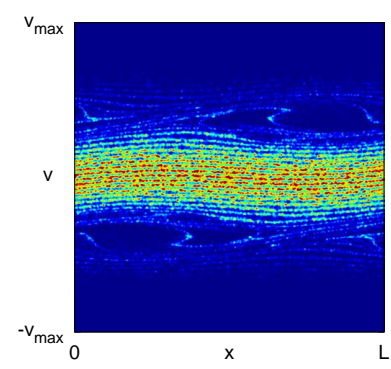

FSL with $\Delta \mathrm{t}_{\mathrm{r}}=2$

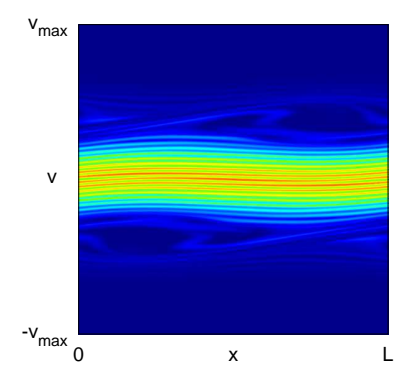

LTPIC with $\Delta \mathrm{t}_{\mathrm{r}}=2$

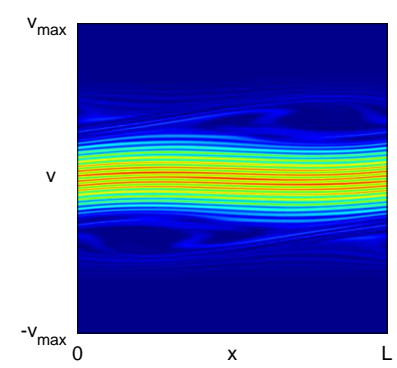

weighted PIC

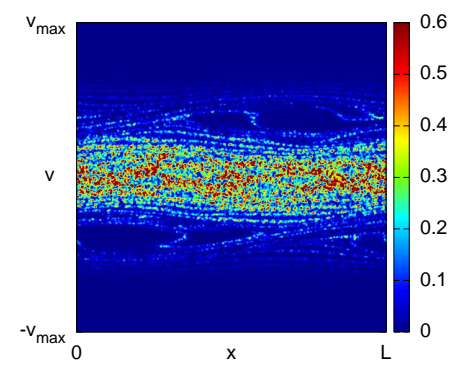

FSL with $\Delta \mathrm{t}_{\mathrm{r}}=4$

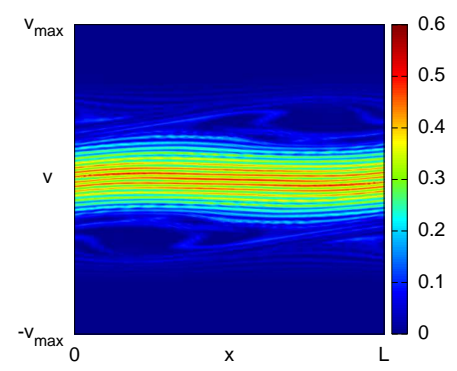

LTPIC with $\Delta \mathrm{t}_{\mathrm{r}}=4$

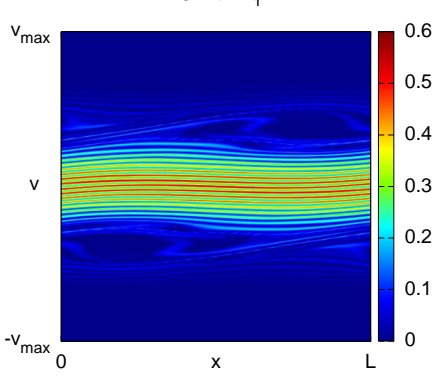

Figure 7: Strong Landau damping. Comparisons of phase-space densities obtained at $t_{n}=60$ with different methods. All the runs use a time step $\Delta t=1 / 8$, a Poisson solver with 256 cells and $256 \times 256$ particles, except for the reference simulation, an LTPIC run with 512 cells and $512 \times 512$ particles. In the FSL and LTPIC runs the remapping period varies as indicated (in the reference run it is $\Delta t_{\mathrm{r}}=4$ ). The approximate cpu times for these runs are $4900 \mathrm{~s}$ (reference LTPIC), $625 \mathrm{~s}$ (unweighted PIC), $650 \mathrm{~s}$ (weighted PIC), 690 to $720 \mathrm{~s}$ (FSL runs) and 655 to $665 \mathrm{~s}$ (LTPIC runs).

Finally, in Figure 8 we show $v$-slices of the distribution at $x=L / 2$ and $t_{n}=60$. Again, results 
show that the LTPIC scheme gives the best results: compared to the PIC method the noise has been removed, and compared to the FSL scheme the numerical diffusion is significantly reduced.

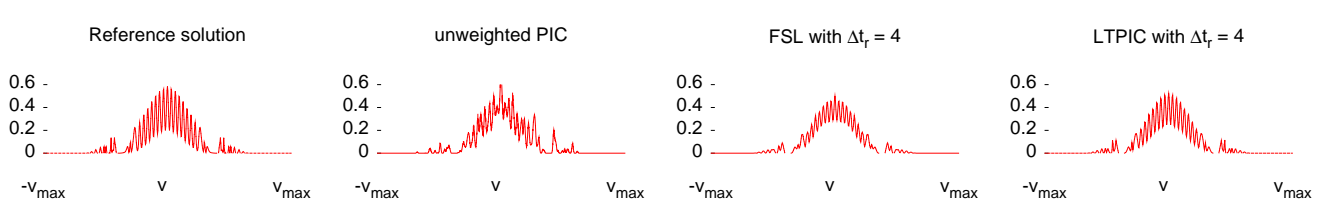

Figure 8: Velocity profiles $f_{h}^{n}(x=L / 2, v)$ at $t_{n}=60$ associated with some of the numerical solutions shown on Figure 7

\subsection{Two-stream instabilities}

Here we consider again the periodic Vlasov-Poisson system and set the initial distribution function as follows.

1. (Weak instability.) First, to compare our results with Refs. [28, 29] we set

$$
f(t=0, x, v) \equiv \frac{2\left(1+5 v^{2}\right)}{7 \sqrt{2 \pi}} e^{-\frac{v^{2}}{2}}\left(1+A\left(\frac{\cos (2 k x)+\cos (3 k x)}{1.2}+\cos (k x)\right)\right)
$$

with $k \equiv \frac{1}{2}$ and a weak amplitude $A \equiv 0.01$ for the perturbation.

2. (Strong instability.) Next to compare our results with Refs. [30, 32] we set

$$
f(t=0, x, v) \equiv \frac{v^{2}}{\sqrt{2 \pi}} e^{-\frac{v^{2}}{2}}(1-A \cos (k x))
$$

with $k \equiv \frac{1}{2}$ and a strong amplitude $A \equiv 0.5$ for the perturbation.

For the simulations we use a cutoff velocity $v_{\max } \equiv 5$ and periodic boundary conditions at $x=0$ and $x=L \equiv 2 \pi / k$.

In Figure 9 we compare phase-space densities for the weak two-stream instability (case 1) obtained at $t=53$ with PIC, FSL and LTPIC runs and various numerical parameters indicated in the figure caption. Again, the results lead to several observations.

- First, LTPIC and FSL are able to remove the "noise" (i.e., the oscillations) for appropriate values of the remapping period $\Delta t_{\mathrm{r}}$. In that regard our simulations show again the robustness of LTPIC compared to FSL, where strong oscillations appear for $\Delta t_{\mathrm{r}} \gtrsim 2$.

- For low remapping periods FSL and LTPIC give similar results - an expected observation since particles have less time to deform. However a closer look at the filaments in the $\Delta t_{\mathrm{r}}=1$ case shows that the latter is less diffusive.

- Again, our measurements indicate that for similar numerical parameters the FSL and LTPIC runs take similar computational time. This signifies that deforming the particles is not an expensive task in our code.

- Finally we find that the LTPIC scheme is able to achieve the accuracy of some highresolution state-of-the-art grid-based methods. For instance, the bottom left panel in Figure 9 showing an LTPIC run using $\Delta t_{\mathrm{r}}=1$ and $128 \times 128$ particles is very similar to the right panel in Figure 11 from Ref. [29], obtained with a conservative third order WENO BSL scheme using a $256 \times 512$ phase-space mesh. 

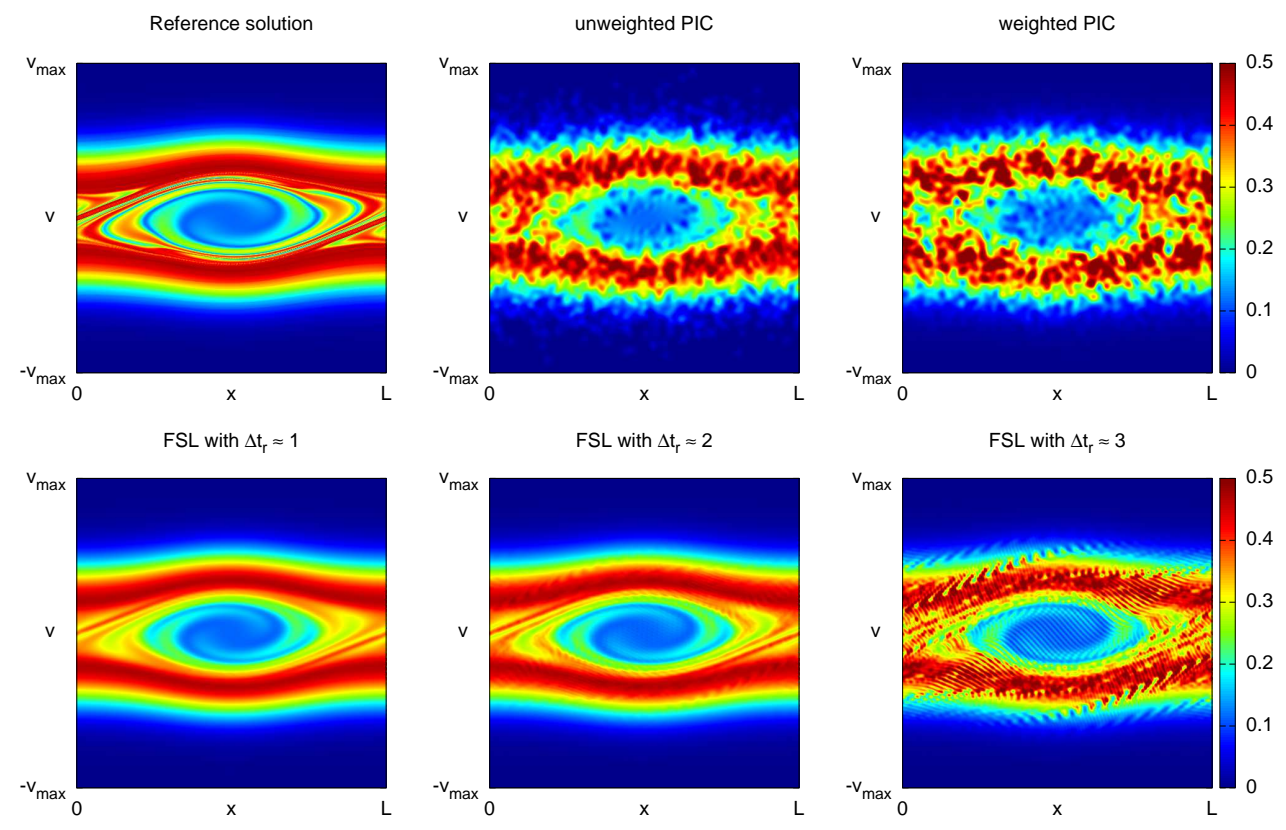

LTPIC with $\Delta \mathrm{t}_{\mathrm{r}} \approx 1$
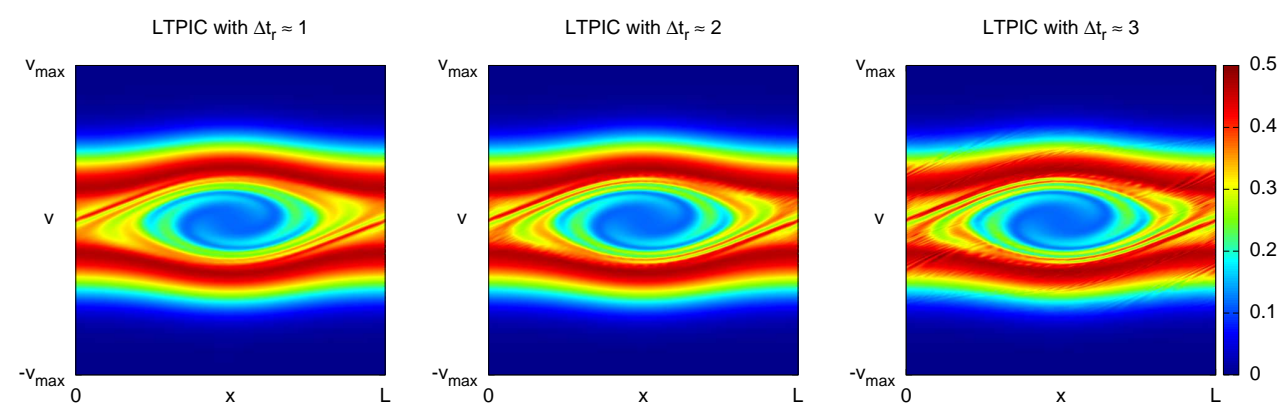

Figure 9: Weak two-stream instability (case 1). Comparisons of phase-space densities obtained at $t_{n}=53$ with different methods. All the runs use a time step $\Delta t \approx 1 / 5$, a Poisson solver with 64 cells and $128 \times 128$ particles, except for the reference simulation, an LTPIC run with 128 cells and $512 \times 512$ particles. In the FSL and LTPIC runs the remapping period varies as indicated (in the reference run it is $\Delta t_{\mathrm{r}} \approx 2$ ). The approximate cpu times for these runs are $875 \mathrm{~s}$ (reference LTPIC), $30 \mathrm{~s}$ (unweighted PIC), $26 \mathrm{~s}$ (weighted PIC), 31 to $37 \mathrm{~s}$ (FSL runs) and 35 to $40 \mathrm{~s}$ (LTPIC runs).

Turning next to the strong two-stream instability (case 2), we show in Figure 10 the time evolution of the phase-space density obtained with an LTPIC run using $256 \times 256$ particles. Fine phase-space detail is resolved as the strong amplitude of the initial perturbation leads to filamentations. Again we can compare our results with high order state-of-the-art grid-based methods. For instance we observe that the bottom right panel in Figure 10 showing the LTPIC density at $t=45$ resolves the filaments with similar accuracy to that of the (center and bottom) panels in Figure 4 from Ref. [32], obtained by a fifth-order BSL-DG scheme using $129 \times 129$ and $255 \times 255$ phase-space cells, respectively. Here some complementary observations are in order. On the one hand indeed, a closer look at the bottom right panel shows that the LTPIC solution presents moderate oscillations in the inner filaments. Therefore, it is not strictly as accurate as the mentionned 
BSL-DG simulations. (To remove these oscillations one may lower the remapping period $\Delta t_{\mathrm{r}}$ but at the cost of more diffusion; one should then use remapping operators less diffusive than the cubic spline quasi-interpolation.) On the other hand, as a forward particle method LTPIC is simpler to implement, and potentially cheaper to run compared to grid-based or BSL methods. We also note that the LTPIC simulation shown in Figure 10 involves significantly larger time steps (namely $\Delta t=1 / 5$ ) than the BSL run where the announced CFL constant corresponds to $\Delta t \approx 1 / 64$. Moreover, as each fifth-order DG cell contains 15 basis functions in $2 \mathrm{~d}$, the $255 \times 255$ BSL-DG run involves approximatively twice as many degrees of freedom as the plotted LTPIC simulation.

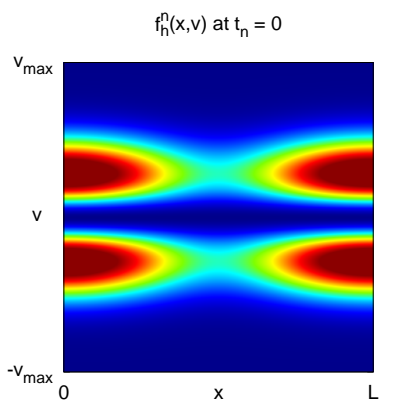

$f_{h}^{n}(x, v)$ at $t_{n}=12$

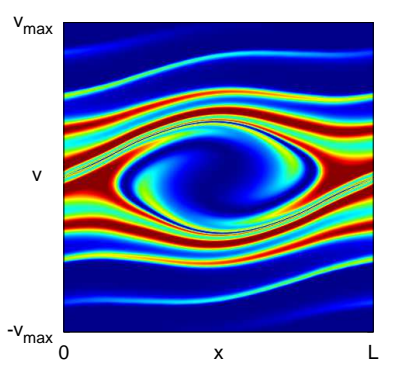

$f_{h}^{n}(x, v)$ at $t_{n}=39$

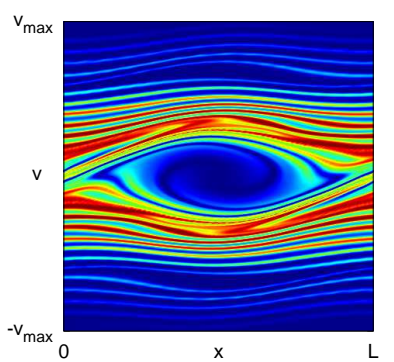

$f_{h}^{n}(x, v)$ at $t_{n}=3$

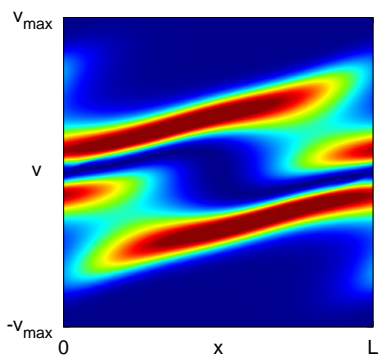

$f_{h}^{n}(x, v)$ at $t_{n}=15$

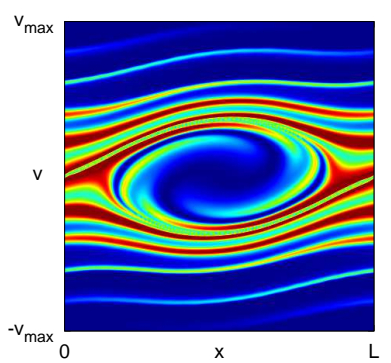

$f_{h}^{n}(x, v)$ at $t_{n}=42$

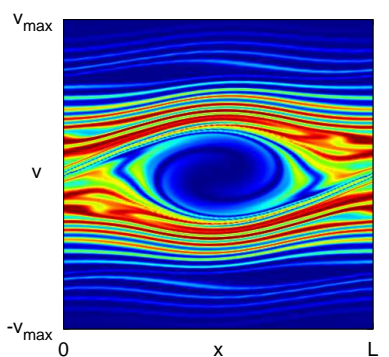

$f_{h}^{n}(x, v)$ at $t_{n}=6$

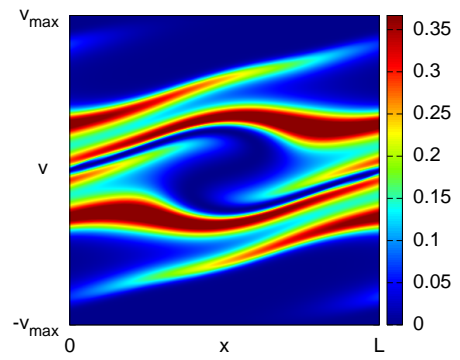

$f_{h}^{n}(x, v)$ at $t_{n}=18$

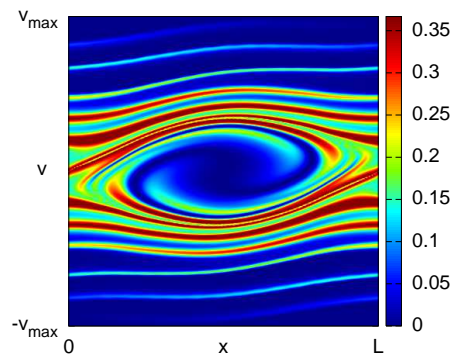

$f_{h}^{n}(x, v)$ at $t_{n}=45$

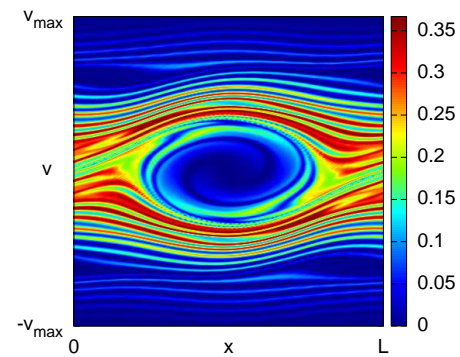

Figure 10: Strong two-stream instability (case 2). Time evolution of the phase-space particle density $f_{h}^{n}(x, v)$ obtained with an LTPIC simulation. This run uses a time step $\Delta t=1 / 5$, a remapping period $\Delta t_{\mathrm{r}} \approx 2$, a Poisson solver with 64 cells and $256 \times 256$ particles. The approximate cpu time for this run is $115 \mathrm{~s}$.

Therefore, in the top row of Figure 11 we show PIC, FSL and LTPIC runs obtained with $512 \times 512$ particles. Now the high-resolution LTPIC run involves about 1.5 as many degrees of 
freedom as the $256 \times 256$ BSL-DG solution shown in Figure 4 from Ref. [32], and it achieves a similar level of details, still with a very large time step. In the bottom row of Figure 11 we then show PIC, FSL and LTPIC runs using $256 \times 256$ particles, for comparison. To highlight the robustness of LTPIC compared to FSL the remapping period is taken higher than in Figure 10. Results indeed show strong ripples in the FSL solutions, but almost none in the LTPIC ones. By running the FSL method with $\Delta t_{\mathrm{r}}=1.8$, we obtain solutions (not shown here) where oscillations are either significantly reduced in the $256 \times 256$ case or fully smoothed out in the $512 \times 512$ case. Finally, our cpu time measurements show that FSL and PIC runs using similar numbers of Poisson cells and particles require very similar computational effort. With the same numerical parameters the LTPIC runs are only slightly longer, which again indicates that the extra work of deforming the particles is not dominant.

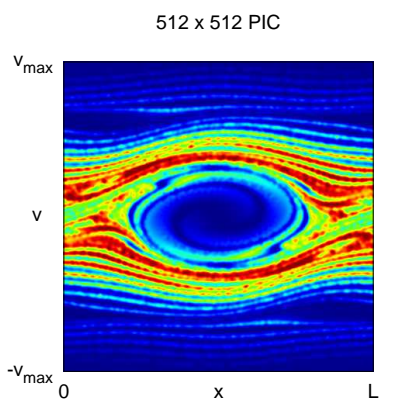

$256 \times 256 \mathrm{PIC}$

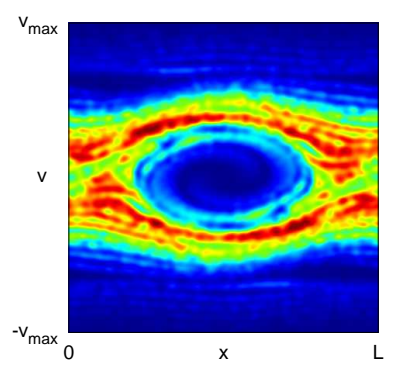

$512 \times 512 \mathrm{FSL}$ with $\Delta \mathrm{t}_{\mathrm{r}}=3$

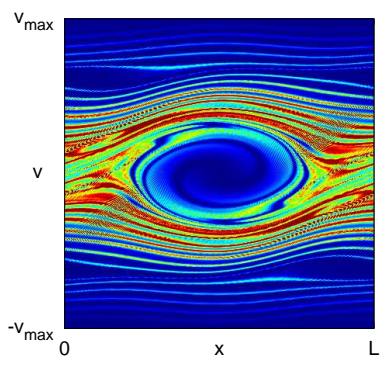

$256 \times 256$ FSL with $\Delta \mathrm{t}_{\mathrm{r}}=3$

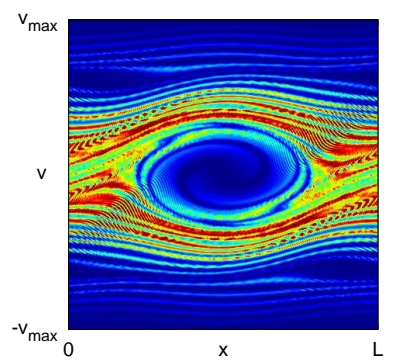

$512 \times 512$ LTPIC with $\Delta \mathrm{t}_{\mathrm{r}}=3$

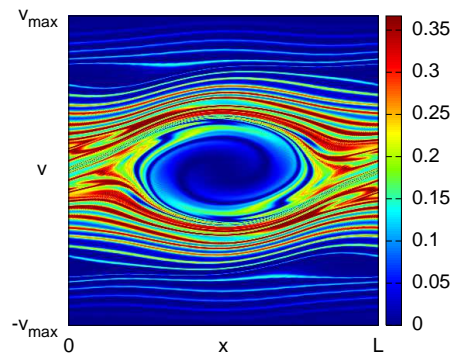

$256 \times 256$ LTPIC with $\Delta \mathrm{t}_{\mathrm{r}}=3$

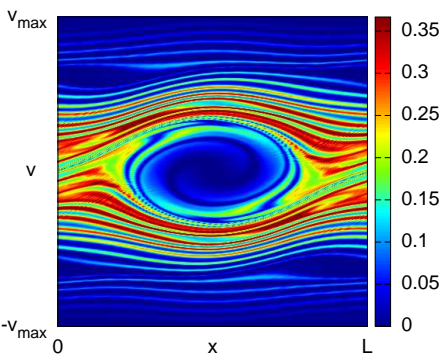

Figure 11: Strong two-stream instability (case 2). Comparisons of phase-space densities obtained at $t_{n}=45$ with high (top) and moderate (bottom) resolution runs. All the runs use a time step $\Delta t=1 / 5$. The top runs use 128 cells and $512 \times 512$ particles, with approximate cpu times $600 \mathrm{~s}$ (PIC), $630 \mathrm{~s}$ (FSL) and $680 \mathrm{~s}$ (LTPIC). The bottom runs use 64 cells and $256 \times 256$ particles, with approximate cpu times $90 \mathrm{~s}$ (PIC), $90 \mathrm{~s}$ (FSL) and $110 \mathrm{~s}$ (LTPIC). Note that the remapping period $\Delta t_{\mathrm{r}}=3$ taken here for the FSL and LTPIC runs is slightly longer than in Figure 10

\subsection{Halo formation in a mismatched thermal sheet beam}

We now consider the case of a 1D sheet beam in a continuous focusing channel with prescribed focusing strength

$$
\kappa(s) \equiv k_{\beta_{0}}^{2},
$$

as studied in Ref. [22]. Here no electron cloud is present $(\kappa$ takes the role of a neutralizing species) and the density $f=f\left(s, x, x^{\prime}\right)$ models an axially thin, transverse slice of a continuous $(\partial / \partial z=\partial / \partial y=0)$ ion beam composed of single species particles of charge $q$ and rest mass $m$. 
The slice propagates with velocity $\beta_{b} c=$ const and relativistic gamma factor $\gamma_{b} \equiv\left(1-\beta_{b}^{2}\right)^{-1 / 2}$ along the axial $(z)$ direction. Here, $c$ is the speed of light in vacuum. The beam phase space is described by the spatial coordinate $x$ and the angle $x^{\prime}$ that the particle trajectories make relative to the longitudinal axis, and the independent timelike coordinate $s$ represents the axial coordinate of a reference particle of the beam (or of the slice being followed), measured along the design orbit (nominally the machine axis). In this model the Vlasov equation reads

$$
\left\{\frac{\partial}{\partial s}+\frac{\partial H}{\partial x^{\prime}} \frac{\partial}{\partial x}-\frac{\partial H}{\partial x} \frac{\partial}{\partial x^{\prime}}\right\} f\left(s, x, x^{\prime}\right)=0
$$

with Hamiltonian

$$
H \equiv \frac{1}{2} x^{\prime 2}+\frac{1}{2} \kappa x^{2}+\frac{q \phi(s, x)}{m \gamma_{b}^{3} \beta_{b}^{2} c^{2}} .
$$

Here, the electrostatic potential $\phi$ is the solution to the Poisson equation

$$
\frac{\partial^{2} \phi}{\partial x^{2}}(s, x)=-\frac{q}{\epsilon_{0}} n_{i}(s, x)
$$

solved with free space boundary conditions $-\frac{\partial \phi}{\partial x}( \pm \infty)= \pm \frac{q}{2 \epsilon_{0}} N_{i}$ to obtain

$$
E(s, x) \equiv-\frac{\partial \phi}{\partial x}(s, x)=\frac{q}{\epsilon_{0}}\left(\int_{-\infty}^{x} n_{i}(s, \tilde{x}) \mathrm{d} \tilde{x}-\frac{1}{2} N_{i}\right)
$$

where $n_{i}(s, x) \equiv \int_{\mathbb{R}} f\left(s, x, x^{\prime}\right) \mathrm{d} x^{\prime}$ is the ion density in configuration space and $N_{i} \equiv \int_{\mathbb{R}} n_{i}(s, x) \mathrm{d} x$ is the integrated ion density, or total number of ions - a constant, as particles are neither created nor destroyed. Following the procedure described therein, we shall first review how thermal equilibrium solutions can be obtained with physical scales roughly consistent with a recent experiment for beam driven Warm Dense Matter called the NDCX-I at Lawrence Berkeley National Laboratory [33]. Specifically, we shall consider a $100 \mathrm{KeV}$ kinetic energy potassium $K^{+}$ion beam (the axial velocity of which can be set nonrelativistically by $m \gamma_{b} \beta_{b}^{2} c^{2} / 2 \approx 10^{5} q$ ) with the sheet beam perveance

$$
P \equiv \frac{q^{2} N_{i}}{2 \epsilon_{0} m \gamma_{b}^{3} \beta_{b}^{2} c^{2}}
$$

to be specified below. Following the analysis carried out in [22], thermal equilibrium distributions can then be obtained as follows. Given a specific value for the positive, dimensionless parameter

$$
\Delta \equiv \frac{\gamma_{b}^{3} \beta_{b}^{2} c^{2} k_{\beta_{0}}^{2}}{\hat{\omega}_{p}^{2}}-1
$$

where $\hat{\omega}_{p} \equiv\left[q^{2} \hat{n} /\left(\epsilon_{0} m\right)\right]^{1 / 2}$ is the plasma frequency formed from the peak density scale $\hat{n}$, a normalized effective potential $\psi_{\Delta}$ is defined as the solution of the transformed Poisson equation

$$
\psi_{\Delta}^{\prime \prime}(\hat{x})=1+\Delta-e^{-\psi_{\Delta}(\hat{x})} \quad \text { with boundary conditions } \quad \psi_{\Delta}^{\prime}(0)=\psi_{\Delta}(0)=0 .
$$

Then, the thermal distribution given by

$$
f^{\mathrm{eq}}\left(x, x^{\prime}\right) \equiv \frac{\hat{n}}{\sqrt{2 \pi T^{*}}} \exp \left(-\frac{x^{\prime 2}}{2 T^{*}}\right) \exp \left(-\psi_{\Delta}\left(\frac{x}{\gamma_{b} \lambda_{D}}\right)\right)
$$


yields a stationary equilibrium solution $f\left(s, x, x^{\prime}\right)=f^{\mathrm{eq}}\left(x, x^{\prime}\right)$ of the Vlasov-Poisson system (32), (33)- 35). Here, $T^{*} \equiv T /\left(m \gamma_{b} \beta_{b}^{2} c^{2}\right)$ is the dimensionless temperature associated with the thermodynamic temperature $T$ (expressed in energy units), and $\lambda_{D} \equiv\left[T /\left(m \hat{\omega}_{p}^{2}\right)\right]^{1 / 2}$ is the corresponding Debye length. We observe that the parameters in (39) can be derived by first inverting (37), i.e.,

$$
\hat{n}=\frac{\epsilon_{0} m \gamma_{b}^{3} \beta_{b}^{2} c^{2} k_{\beta_{0}}^{2}}{q^{2}(1+\Delta)},
$$

and next infering from (36) and $N_{i}=\int_{-\infty}^{\infty} n_{i}(x) \mathrm{d} x=2 \hat{n} \gamma_{b} \lambda_{D} \int_{0}^{\infty} e^{-\psi_{\Delta}(\hat{x})} \mathrm{d} \hat{x}$ that

$$
\gamma_{b} \lambda_{D}=\frac{N_{i}}{2 \hat{n} \int_{0}^{\infty} e^{-\psi_{\Delta}(\hat{x})} \mathrm{d} \hat{x}}=\frac{P}{k_{\beta_{0}}^{2}} \frac{(1+\Delta)}{\int_{0}^{\infty} e^{-\psi_{\Delta}(\hat{x})} \mathrm{d} \hat{x}} .
$$

The resulting temperature is then

$$
T^{*}=\left(\frac{P}{k_{\beta_{0}}}\right)^{2} \frac{1+\Delta}{\left(\int_{0}^{\infty} e^{-\psi_{\Delta}(\hat{x})} \mathrm{d} \hat{x}\right)^{2}} .
$$

The tune depression $\sigma / \sigma_{0}$ - defined as the ratio between the phase advance of the particles in the presence and absence of beam charge - can be calculated [22] as

$$
\sigma / \sigma_{0}=\left[1-\frac{1}{\sqrt{3}(1+\Delta)} \frac{\left(\int_{0}^{\infty} e^{-\psi_{\Delta}(\hat{x})} \mathrm{d} \hat{x}\right)^{3 / 2}}{\left(\int_{0}^{\infty} \hat{x}^{2} e^{-\psi_{\Delta}(\hat{x})} \mathrm{d} \hat{x}\right)^{1 / 2}}\right]^{\frac{1}{2}} .
$$

By solving numerically (38), (41), it is then possible to prescribe a specific tune depression and derive the corresponding value of $\Delta$ to specify the equilibrium distribution: the resulting parameters are given in [22, Table II] for regularly spaced values of $\sigma / \sigma_{0} \in\{0.1,0.2, \ldots, 0.9\}$, and in Table 1 below for a strong tune depression, i.e. $\sigma / \sigma_{0}=0.1$. For the purpose of comparing our results to typical NDCX-I experiments, we set the focusing strength to $k_{\beta_{0}} \equiv \sigma_{0} / L_{p}$ in such a way that free particles have a phase advance of $\sigma_{0} \sim \pi / 3$ per lattice period $L_{p}=0.5 \mathrm{~m}$, and we set the perveance by taking $P / k_{\beta_{0}}=0.01$. We note that for a sheet beam, the perveance has dimension $1 /$ length and $P / k_{\beta_{0}}$ is dimensionless. The distribution corresponding to $\sigma / \sigma_{0}=0.1$ corresponds to a highly nonlinear form in $x$ due to the radial beam extent and the nonlinear solution for the effective potential $\psi_{\Delta}$.

\begin{tabular}{cccccc}
\hline \hline depression & parameter & temperature & Debye length & rms radius & peak density \\
$\sigma / \sigma_{0}$ & $\Delta$ & $T^{*}$ & $\lambda_{D}$ & $x_{b}^{\text {eq }}$ & $\hat{n}$ \\
\hline 0.1 & $5.522 \times 10^{-8}$ & $3.463 \times 10^{-7}$ & $2.810 \times 10^{-4} \mathrm{~m}$ & $4.822 \times 10^{-3} \mathrm{~m}$ & $4.848 \times 10^{13}$ \\
\hline \hline
\end{tabular}

Table 1: Physical parameters for the matched thermal sheet beams with $100 \mathrm{KeV} K^{+}$ions, corresponding to an axial beam velocity of $\beta_{b} c$ with $\beta_{b}=2.343 \times 10^{-3}$ and relativistic factor $\gamma_{b} \approx 1$. We take $k_{\beta_{0}} \approx 2.094$ corresponding to a $60^{\circ}$ phase advance per lattice period $L_{p}=0.5 \mathrm{~m}$, and the perveance is set to $P=0.01 k_{\beta_{0}}$.

Finally, from (39) we derive an initially "mismatched" beam through a canonical transformation that dilates the distribution in the spatial dimension while preserving its perveance and initial effective phase-space area (emittance) by taking

$$
f\left(s=0, x, x^{\prime}\right) \equiv f_{22}^{\mathrm{eq}}\left(\frac{x}{\mu}, \mu x^{\prime}\right), \quad \mu>0 .
$$


Here $\mu$ corresponds to the mismatch parameter, defined as the ratio of the initial (rms) beam radius to the radius of the matched beam, see e.g. Ref. [34].

In Figure 12 we show the evolution of a mismatched beam with a thermal equilibrium form specified by (39) using the procedure outlined above with $\mu=1.25$ and a tune depression of $\sigma / \sigma_{0}=0.1$. Here the numerical solution is computed with an LTPIC scheme on a computational domain corresponding to $|x| \leq 15 \mathrm{~mm}$ and $\left|x^{\prime}\right| \leq 14.5 \mathrm{mrad}$. In the phase-space plots we vizualise the tenuous halo that evolves from the initial distribution by taking contours of the numerical density using exponential increments. Filled color contours illustrate the core of the phase-space density.

In Figure 13 we next compare the phase-space densities at $s=20 \mathrm{~m}$ with halo contours obtained with different schemes using $256 \times 256$ particles. Here we observe that the unweighted PIC has a low level of noise in the core but misses almost all of the halo. The weighted PIC simulation catches a fair proportion of the halo but in a very fragmented way, and in addition it has a high level of noise in the core. In contrast, the FSL simulation with short remapping periods (shown on the center left panel) does a reasonable job, although it still misses some part of the halo arms. For longer remapping periods (center and center right panels) it is severly hampered by phase-space oscillations. In the LTPIC simulations (bottom panels) these numerical artifacts are significantly reduced, which shows once more the ability of this new approach to remove the noise at reasonable computational cost, and with similar or improved accuracy.

\section{Conclusion}

We have presented a new deterministic PIC method for electrostatic plasma simulations, wherein finite-sized particles have a shape function that is linearly transformed in time to approximately follow the flow and thereby reduce the oscillations traditionally observed in standard PIC simulations. Although this method may be seen as an extension of the remapped-particle (FSL) scheme [8] (due to the practical need to remap the particles after the flow has evolved significantly), its relative robustness to low remapping frequencies makes it actually closer to a proper particle scheme. By testing our method on benchmarked test cases we have demonstrated its ability to effectively reduce the noise and reach accuracy levels similar to those of expensive high order state-of-the-art Vlasov schemes.

Simulations of real-world systems often need to capture the production of phase space structures by collective interactions. For example, in accelerator physics, beam halo must be minimized in order to limit the unwanted particle loss on machine surfaces. The simulated halo location and density must be quantitatively correct, in a calculation wherein the far denser core of the distribution must also be evolved self-consistently (since the core fields influence the halo dynamics). On test problems of this type the LTPIC method performs well, and we believe that it has considerable promise to augment the standard PIC methods predominately employed today.

\section{Acknowledgments}

Most of this work was carried out in 2010-2011 while Martin Campos Pinto was a Fulbright visiting scholar at the Lawrence Berkeley National Laboratory with partial support from the France-Berkeley Fund. Fruitful interactions with John Verboncoeur are gratefully acknowledged. The work of the final three authors was performed under the auspices of the USDOE by LLNL under Contract DE-AC52-07NA27344. 

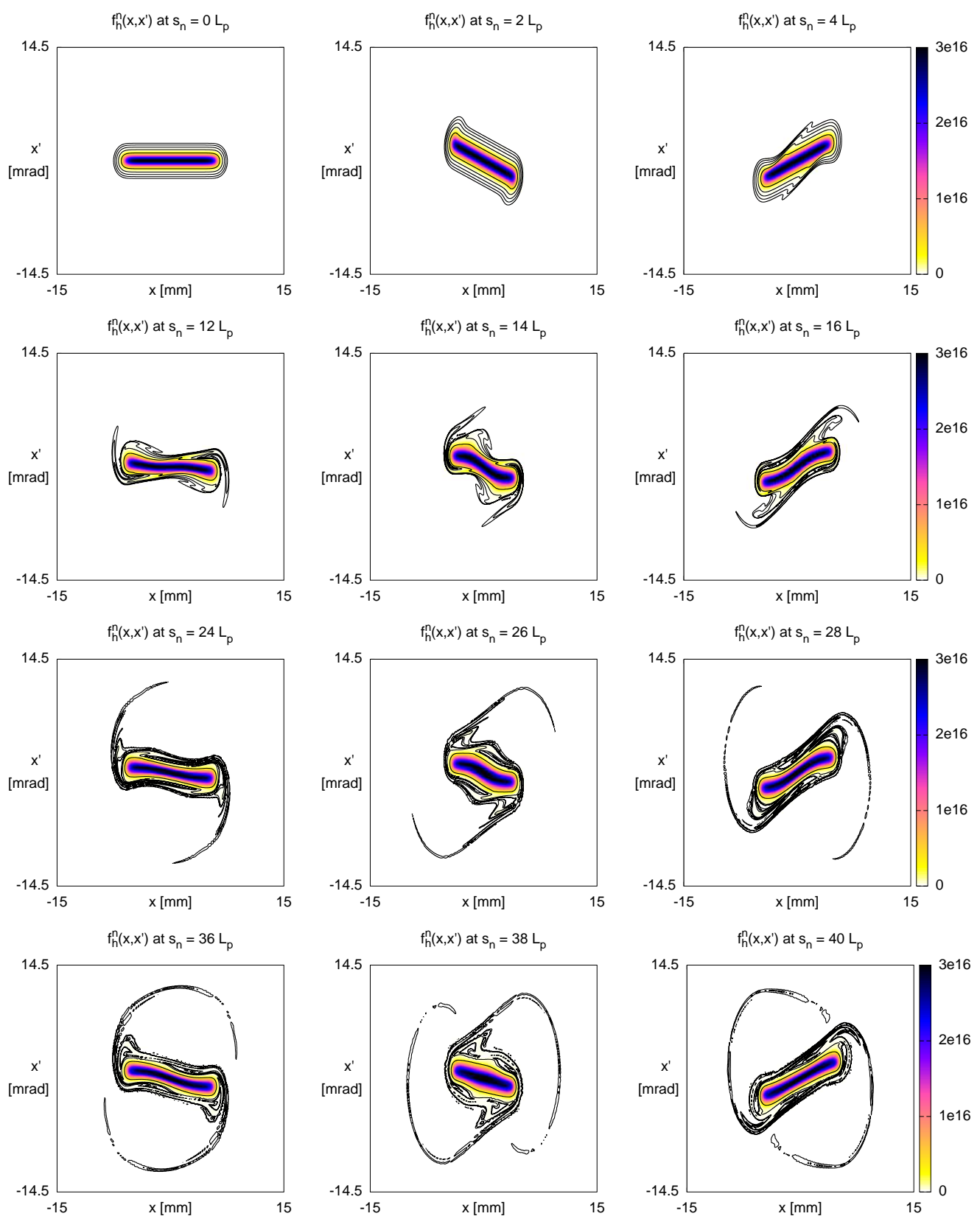

Figure 12: Mismatched thermal sheet beam in a continuous focusing lattice with phase advance of $60^{\circ}$ per period $L_{p}=0.5 \mathrm{~m}$, and tune depression $\sigma / \sigma_{0}=0.1$. The plots show the evolution of the phase-space density (with respect of the timelike, longitudinal coordinate $s$ ) obtained with an LTPIC simulation. This run uses a time step $\Delta s=L_{p} / 16$, a remapping period $\Delta s_{\mathrm{r}}=2.5 L_{p}$, a Poisson solver with 128 cells and $256 \times 256$ particles. The halo is shown through isolines corresponding to values of $10^{-1}, 10^{-2}, \ldots, 10^{-5}$ of the peak initial density. The approximate cpu time for this run is $220 \mathrm{~s}$. 

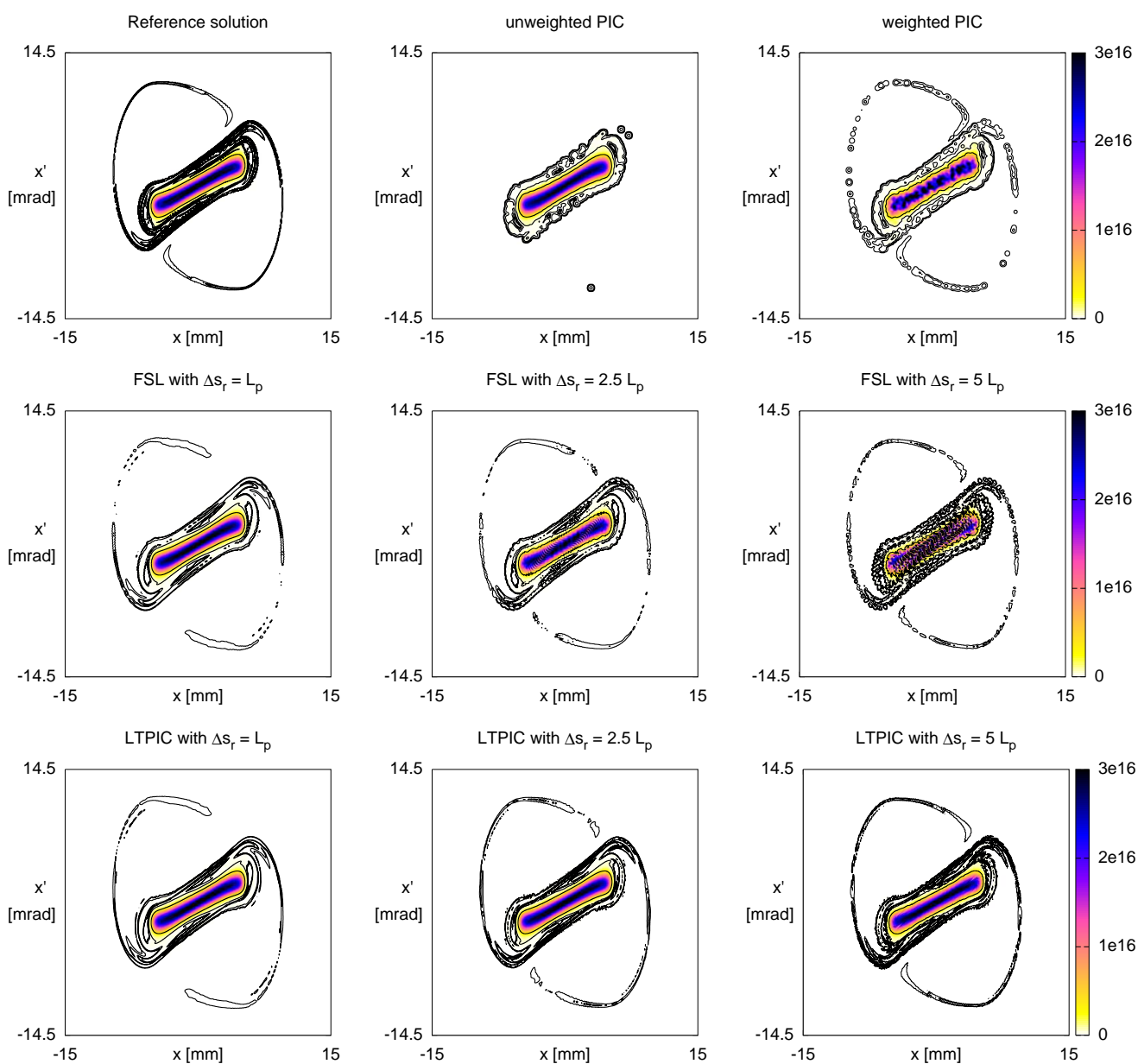

Figure 13: Mismatched thermal beam. Comparisons of phase-space densities obtained at $s_{n}=40 L_{p}$ with different methods. All the runs use a time step $\Delta s=L_{p} / 16$, a Poisson solver with 128 cells and $256 \times 256$ particles, except for the reference simulation, an LTPIC run with 256 cells and $512 \times 512$ particles. In the FSL and LTPIC runs the remapping period varies as indicated (in the reference run it is $\Delta s_{\mathrm{r}}=2.5 L_{p}$ ). The approximate cpu times for these runs are $1450 \mathrm{~s}$ (reference LTPIC), $225 \mathrm{~s}$ (unweighted PIC), $145 \mathrm{~s}$ (weighted PIC), 220 to $265 \mathrm{~s}$ (FSL runs) and 200 to $245 \mathrm{~s}$ (LTPIC runs). 


\section{References}

\section{References}

[1] J. T. Beale, A. Majda, Vortex methods. II. Higher order accuracy in two and three dimensions, Mathematics of Computation 39 (1982) 29-52.

[2] P.-A. Raviart, An analysis of particle methods, in: Numerical methods in fluid dynamics (Como, 1983), Lecture Notes in Mathematics, Berlin, 1985, pp. 243-324.

[3] A. Ghizzo, P. Bertrand, M. Shoucri, T. Johnston, E. Fijalkow, M. Feix, V. Demchenko, Study of laser-plasma beat wave current drive with an Eulerian Vlasov code, Nuclear Fusion 32 (1992) 45-65.

[4] M. Shoucri, A. Cardinali, J. Matte, R. Spigler, Numerical study of plasma-wall transition using an Eulerian Vlasov code, The European Physical Journal D 30 (2004) 81-92.

[5] F. Valsaque, G. Manfredi, Numerical study of plasma-wall transition in an oblique magnetic field, Journal of nuclear materials 290-293 (2001) 763-767.

[6] E. Sonnendrücker, J. J. Barnard, A. Friedman, D. P. Grote, S. M. Lund, Simulation of heavy ion beams with a semi-Lagrangian Vlasov solver, Nuclear Instruments and Methods in Physics Research A 464 (2001) 470-476.

[7] M. V. Goldman, D. L. Newman, P. Pritchett, Vlasov simulations of electron holes driven by particle distributions from PIC reconnection simulations with a guide field, Geophysical Research Letters 35 (2008) L22109.

[8] J. Denavit, Numerical simulation of plasmas with periodic smoothing in phase space, Journal of Computational Physics 9 (1972) 75-98.

[9] R. D. Nair, J. S. Scroggs, F. H. M. Semazzi, A forward-trajectory global semi-Lagrangian transport scheme, Journal of Computational Physics 190 (2003) 275-294.

[10] N. Crouseilles, T. Respaud, E. Sonnendrücker, A forward semi-Lagrangian method for the numerical solution of the Vlasov equation, Computer Physics Communications 180 (2009) 1730-1745.

[11] M. Bergdorf, P. Koumoutsakos, A lagrangian particle-wavelet method, Multiscale Model. Simul. 5 (2006) 980995.

[12] B. Wang, G. Miller, P. Colella, A Particle-In-Cell method with adaptive phase-space remapping for kinetic plasmas, SIAM Journal on Scientific Computing 33 (2011) 3509-3537.

[13] J.-P. Chehab, A. Cohen, D. Jennequin, J. Nieto, C. Roland, J. Roche, An adaptive particle-in-cell method using multi-resolution analysis, in: Numerical methods for hyperbolic and kinetic problems, volume 7 of IRMA Lect. Math. Theor. Phys., Eur. Math. Soc., Zürich, 2005, pp. 29-42.

[14] B. Terzic, I. Pogorelov, Wavelet-Based Poisson Solver for Use in Particle-in-Cell Simulations, Annals of the New York Academy of Sciences 1045 (2005) 55-67.

[15] S. Gassama, E. Sonnendrücker, K. Schneider, M. Farge, M. Domingues, Wavelet denoising for postprocessing of a 2D Particle-In-Cell code, ESAIM: Proceedings 16 (2007) 195-210.

[16] M. Campos Pinto, Smooth particle methods without smoothing, arXiv:1112.1859 (to be submitted for publication) (2012) 1-32.

[17] T. Hou, Convergence of a Variable Blob Vortex Method for the Euler and Navier-Stokes Equations, SIAM Journal on Numerical Analysis 27 (1990) 1387-1404

[18] A. Cohen, B. Perthame, Optimal Approximations of Transport Equations by Particle and Pseudoparticle Methods, SIAM Journal on Mathematical Analysis 32 (2000) 616-636.

[19] W. Bateson, D. Hewett, Grid and Particle Hydrodynamics, Journal of Computational Physics 144 (1998) 358-378.

[20] D. Hewett, Fragmentation, merging, and internal dynamics for PIC simulation with finite size particles, Journal of Computational Physics 189 (2003) 390-426.

[21] C. Alard, S. Colombi, A cloudy Vlasov solution, Monthly Notices of the Royal Astronomical Society 359 (2005) $123-163$.

[22] S. M. Lund, A. Friedman, G. Bazouin, Sheet beam model for intense space charge: Application to Debye screening and the distribution of particle oscillation frequencies in a thermal equilibrium beam, Phys. Rev. Special Topics Accelerators and Beams 14 (2011) 054201.

[23] C. de Boor (Ed.), A Practical Guide to Splines, Springer-Verlag, New York, 1978.

[24] C. Chui, H. Diamond, A Characterization of Multivariate Quasi-interpolation Formulas and its Applications, Numerische Mathematik 57 (1990) 105-121.

[25] M. Unser, I. Daubechies, On the approximation power of convolution-based least squares versus interpolation, IEEE Transactions on Signal Processing 45 (1997) 1697-1711.

[26] R. Hockney, J. Eastwood, Computer simulation using particles, Taylor \& Francis, Inc, Bristol, PA, USA, 1988.

[27] T. Nakamura, T. Yabe, Cubic interpolated propagation scheme for solving the hyper-dimensional Vlasov-Poisson equation in phase space, Computer Physics Communications 120 (1999) 122-154.

[28] F. Filbet, E. Sonnendrücker, Comparison of Eulerian Vlasov solvers, Computer Physics Communications 150 (2003) 247-266. 
[29] J.-M. Qiu, A. Christlieb, A conservative high order semi-Lagrangian WENO method for the Vlasov equation, Journal of Computational Physics 229 (2010) 1130-1149.

[30] J. Banks, J. Hittinger, A New Class of Nonlinear Finite-Volume Methods for Vlasov Simulation, IEEE Transactions on Plasma Science 38 (2010) 2198-2207.

[31] N. Crouseilles, M. Mehrenberger, F. Vecil, Discontinuous Galerkin semi-Lagrangian method for Vlasov-Poisson, ESAIM: Proceedings 32 (2011) 211-230.

[32] J. Rossmanith, D. Seal, A positivity-preserving high-order semi-Lagrangian discontinuous Galerkin scheme for the Vlasov-Poisson equations, Journal of Computational Physics 230 (2011) 6203-6232.

[33] F. Bieniosek, E. Henestroza, M. Leitner, B. Logan, R. More, P. Roy, P. Ni, P. Seidl, W. Waldron, J. Barnard, High-energy density physics experiments with intense heavy ion beams, Nuclear Instruments and Methods in Physics Research Section A: Accelerators, Spectrometers, Detectors and Associated Equipment 606 (2009) 146151. Heavy Ion Inertial Fusion - Proceedings of the 17th International Symposium on Heavy Ion Inertial Fusion.

[34] T. Wangler, R. Garnett, E. Gray, R. Ryne, T. Wang, Dynamics of beam halo in mismatched beams, in: XVIII International Linac Conference, Genève, Suisse, IEEE \#01CH37268C, Piscataway, NJ 08855, 1996, p. TPAT061. 